\title{
A transcriptomics-guided drug target discovery strategy identifies novel receptor ligands for lung regeneration
}

4 Xinhui $\mathrm{Wu}^{1,2}$, I. Sophie T. Bos ${ }^{1,2}$, Thomas M. Conlon ${ }^{3}$, Meshal Ansari ${ }^{3}$, Vicky 5 Verschut ${ }^{1,6}$ Lars A. Verkleij ${ }^{1,2}$, Angela D’Ambrosi ${ }^{1,2}$, Aleksey Matveyenko ${ }^{4}$, Herbert B. 6 Schiller $^{3}$, Melanie Königshoff ${ }^{5}$, Martina Schmidt ${ }^{1,2}$, Loes E. M. Kistemaker ${ }^{1,2,6}$, Ali 7 Önder Yildirim ${ }^{3}$, Reinoud Gosens , $^{1,2}$ *

$9{ }^{1}$ Department of Molecular Pharmacology, Faculty of Science and Engineering, University of Groningen, 10 Antonius Deusinglaan 1, 9713AV, Groningen, The Netherlands, ${ }^{2}$ Groningen Research Institute for 11 Asthma and COPD, University Medical Center Groningen, University of Groningen, Groningen, The 12 Netherlands, ${ }^{3}$ Institute of Lung Biology and Disease (ILBD)/Comprehensive Pneumology Center (CPC), 13 Helmholz Zentrum München, Munich, Germany, Member of the German Center for Lung Research 14 (DZL), " Department of Physiology and Biomedical Engineering, Mayo Clinic Collage of Medicine, 15 Rochester, Minnesota, USA, ${ }^{5}$ University of Pittsburg, ${ }^{6}$ Aquilo BV, Groningen, The Netherlands, $16{ }^{*}$ Correspondence 


\section{Abstract}

20 Currently, there is no pharmacological treatment targeting defective tissue repair in

21 chronic disease. Here we utilized a transcriptomics-guided drug target discovery strategy using gene signatures of smoking-associated chronic obstructive pulmonary disease (COPD) and from mice chronically exposed to cigarette smoke, identifying

24 druggable targets expressed in alveolar epithelial progenitors of which we screened 25 the function in lung organoids. We found several drug targets with regenerative potential of which EP and IP prostanoid receptor ligands had the most significant

27 therapeutic potential in restoring cigarette smoke-induced defects in alveolar epithelial progenitors in vitro and in vivo. Mechanistically, we discovered by using scRNAsequencing analysis that circadian clock and cell cycle/apoptosis signaling pathways were enriched in alveolar epithelial progenitor cells in COPD patients and in a relevant model of COPD, which was prevented by PGE2 or PGI2 mimetics. Conclusively, specific targeting of EP and IP receptors offers therapeutic potential for injury to repair in COPD.

Key words: PGE2; PGI2; repair; cell cycle; circadian clock signaling; drug screening 


\section{Introduction}

40 One of the main challenges in pharmacology today is the generation of drugs with regenerative potential, with the ability to restore tissue repair in chronic disease. Regenerative medicine has thus far mainly focused on transplantation, tissue engineering approaches, stem- or progenitor cell therapy, or a combination of these ${ }^{1}$.

A regenerative pharmacological approach will have considerable additional potential because it can be applied on a relatively large scale. Furthermore, it can be used to halt the disease process in an early stage resulting in real disease-modifying treatment. Also, pharmacological targeting may aid or support other regenerative strategies. neurological diseases as well as many other disease areas. In respiratory medicine, chronic obstructive pulmonary disease (COPD) is one of the most common lung diseases with a need for regenerative therapies. The disease is characterized by airflow limitation that is not fully reversible, and which deteriorates progressively. The main difficulty underlying COPD pathogenesis is increased tissue destruction in combination with abnormal tissue repair in susceptible individuals. As current therapies do not modify the course of the disease, developing new therapeutic strategies aiming at regeneration of tissue is necessary.

In affected individuals, there is an increase in alveolar air space associated with destruction of alveolar epithelial cells along with reduced capacity of epithelial progenitors to restore this defect. In the distal lung, alveolar type II cells and alveolar epithelial progenitors harbour stem cell capacity and function to maintain alveolar 61 epithelium ${ }^{2}$. These cells reside in a local tissue microenvironment called the niche, which is composed of supporting cells such as fibroblasts and alveolar macrophages.

63 The niche controls adequate activation of the progenitor cell ${ }^{1-3}$ by means of secreted 
64 factors such as WNTs, FGFs, retinoic acid, and many other factors that control stemness, proliferation and differentiation ${ }^{3}$.

Like in many chronic diseases associated with ageing, this local lung microenvironment is insufficiently supportive for lung repair in COPD ${ }^{1,4,5}$. For example, studies have indicated that an imbalance of canonical and noncanonical WNT signaling results in impaired alveolar regeneration in COPD ${ }^{4,6}$. Moreover, lymphotoxin$\beta(L T \beta)$, released from CD8+ T-cells in COPD, can negatively interfere with repair. LT $\beta$ induces noncanonical NFKB signalling, thereby repressing functional Wnt/ $\beta$ catenin signalling in the lung ${ }^{5}$. Accordingly, the challenge towards successful regenerative pharmacology in COPD needs to take into consideration the specific hostile microenvironment and the abnormal repair process that stands in the way of adequate regeneration in COPD.

In the present study, we hypothesized that a transcriptomics guided drug target discovery strategy based on gene signatures differentially expressed in COPD and in response to cigarette smoke may be used to identify novel druggable gene targets that are specifically involved in defective lung repair in COPD. Our results show that such a strategy coupled to functional studies in organoids yields novel receptor ligands of which EP and IP prostanoid receptor had the most significant potential in counteracting the negative effects of cigarette smoke on alveolar progenitor cell function.

\section{Results}

Transcriptomics-guided screening to identify novel targets. We set out to identify novel drug targets that may help restore defective lung repair. To achieve this, we utilized a transcriptomics-guided target discovery strategy (described in Fig. 1a) based on gene signatures of COPD lung tissues ${ }^{7}$ and of a relevant model of cigarette smoke 
exposure $^{8}$ to identify differentially regulated druggable genes. We found Reactome pathways related to inflammation such as Neutrophil degranulation and Innate immune system as well as pathways related to the extracellular matrix such as Extracellular matrix organization and Integrin cell surface interactions to be enriched in both datasets (Fig. 1b-e). We identified 38 individual target genes that were concordantly up- and 30 individual target genes concordantly downregulated. These genes were filtered through the 'Drug-gene interactions and potential druggability in the drug gene interaction database' (DGIdb, http://www.dgidb.org/), which rendered 25 druggable upregulated genes and 16 druggable downregulated genes (Fig. 1f). Genes were further filtered for expression in lung epithelial cells or fibroblasts by consulting the human lung cell atlas (https://asthma.cellgeni.sanger.ac.uk/) and lung map (https://lungmap.net/), which yielded 15 druggable target genes.

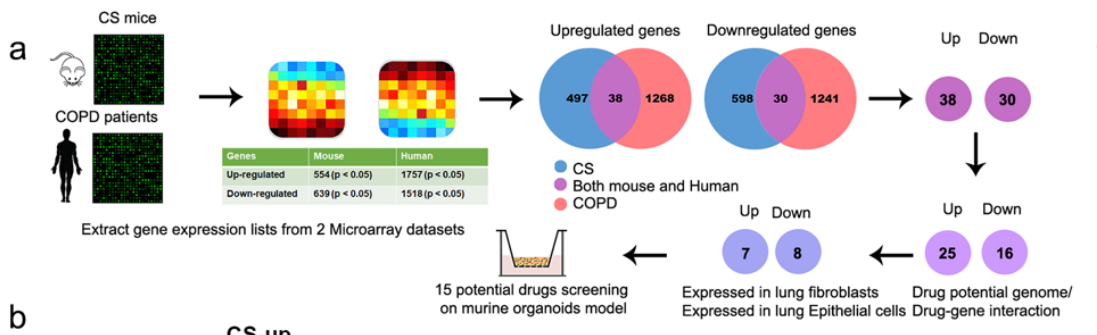

b CS-up

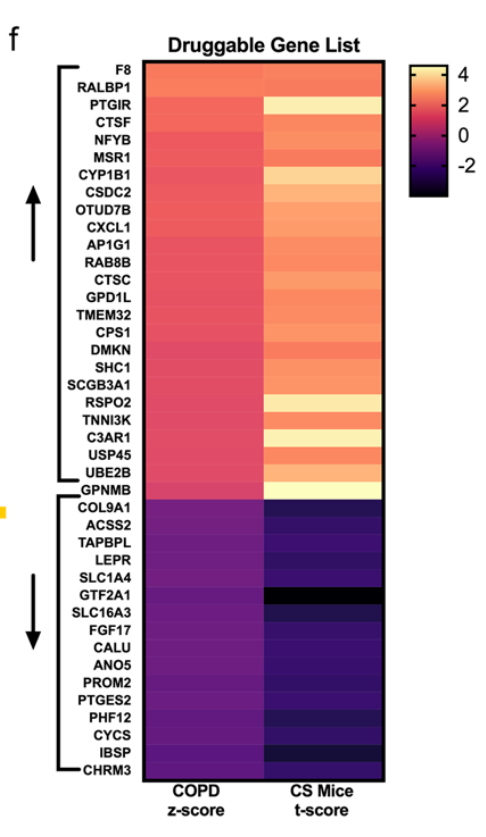

102 Figure 1 Overview of the transcriptomics-guided drug discovery strategy. a Schematic outline of

103 the drug screening strategy. b-c Reactome pathway enrichment analysis of differentially up- and 104 downregulated genes from CS-exposed mice ${ }^{8}$ using GSEA (https://www.gsea- 
106 downregulated genes from COPD patients ${ }^{7}$ using GSEA, the top 20 pathways enriched are presented.

107 f Heatmap shows the gene expression pattern of the druggable genes (http://www.dgidb.org/) identified

108 both in CS-exposed mice and COPD patient databases.

109 To assess the potential relevance of signaling functionally associated with the 15

110 genes of interest, we set up an in vitro organoid model to perform specific drug

111 screening tests. We co-cultured human and mouse CD31-/CD45-/ EpCAM ${ }^{+}$lung

112 epithelial cells with CCL206 lung fibroblasts in organoids in Matrigel and exposed

113 these in vitro to different concentrations $(1.25 \%, 2.5 \%, 5 \%)$ of cigarette smoke extract

114 (CSE) (Fig. 2a, c). The number and size of organoids established by co-culturing

115 human lung tissue derived CD31-/CD45-/EpCAM ${ }^{+}$cells and MRC5 fibroblasts was

116 significantly decreased by CSE in a concentration dependent manner at day 14 (Fig.

$1172 \mathrm{~b})$. The total number of murine organoids quantified at day 14 of treatment with

118 different concentrations of CSE yielded similar results and was decreased in a CSE

119 dose dependent manner as well (Fig. 2e). To specifically analyze the impact of CSE

120 on organoids derived from alveolar epithelial progenitors, we morphologically

121 subclassified organoids into airway and alveolar type ${ }^{9}$ organoids (Fig. $2 \mathrm{~d}$ ), which revealed that alveolar organoid numbers were more susceptible to cigarette smoke extract exposure than airway organoids (Fig. 2e). Immunofluorescence (IF) studies confirmed that the number of acetylated- $\alpha$ tubulin+ (ACT; ciliated cell marker, airway

125 type organoids) and pro-SPC ${ }^{+}$(type II cell marker, alveolar type organoids) organoids

126 was significantly decreased by $5 \%$ CSE (Fig. 2f). The size of both organoid types was 127 decreased at day 14 (Fig. 2g). 
a

Experimental Design (in vitro)

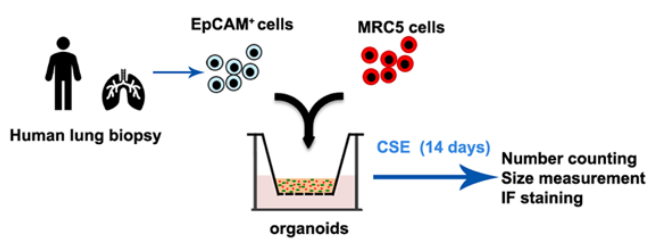

C

Experimental Design (in vitro)

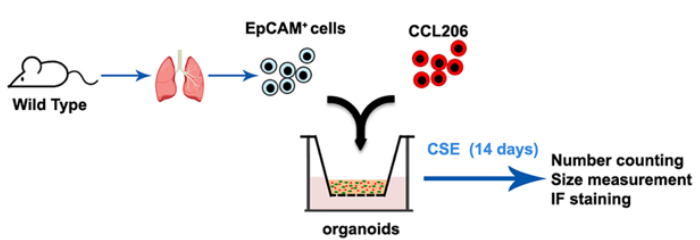

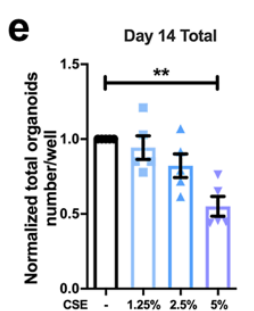

h
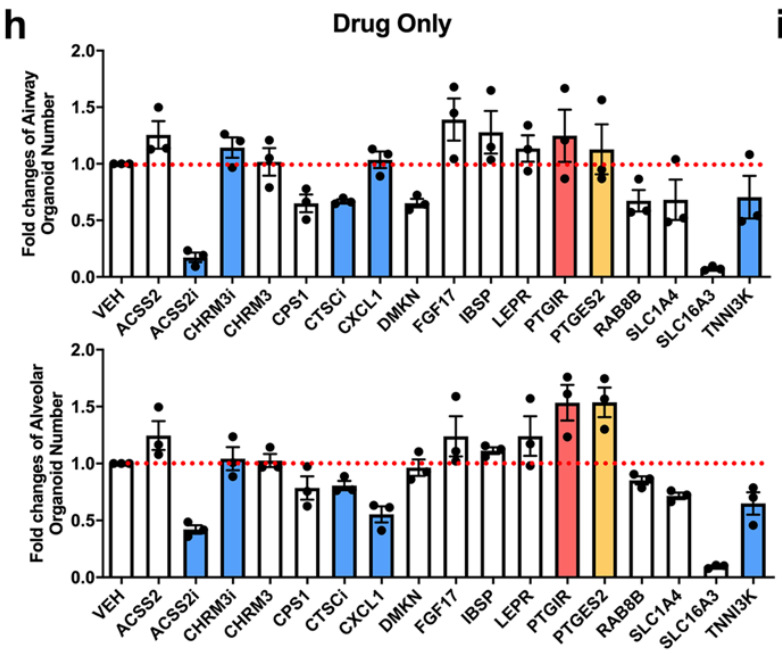

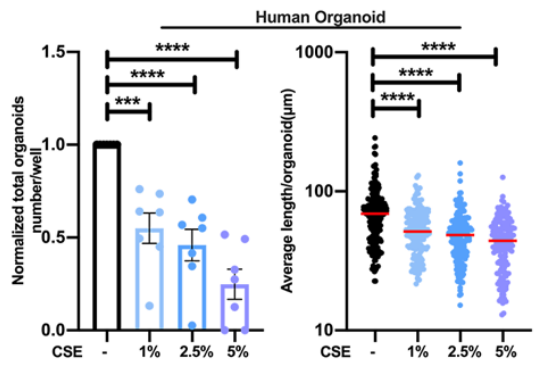

d

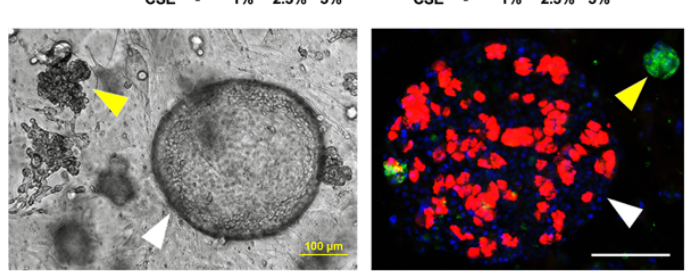

b

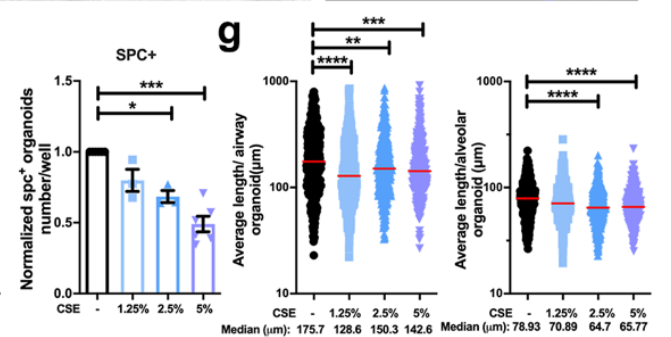

i

$5 \%$ CSE + Drug
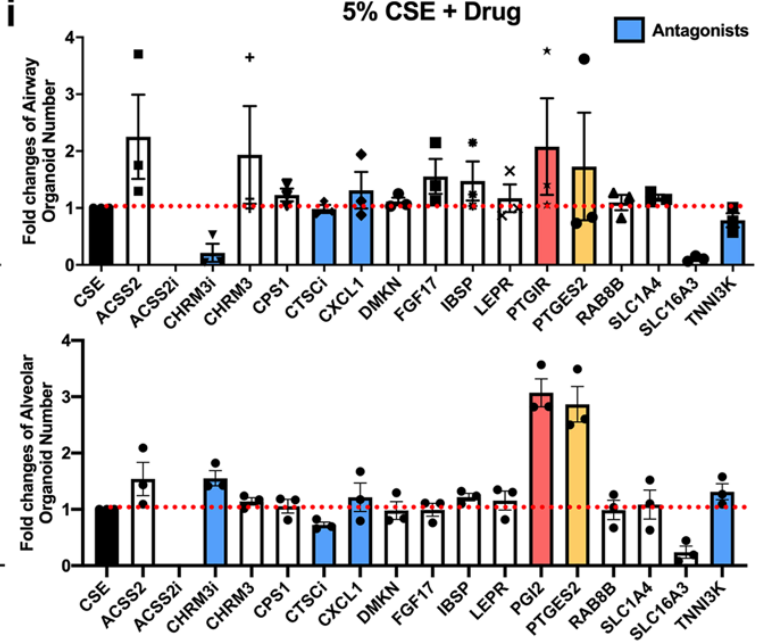

129 Figure 2. Cigarette smoke exposure represses adult epithelial lung organoid formation. a

130 Schematic of in vitro human experimental design. b Quantification of total amount of human organoids

131 and the quantification of average human organoid diameters after treatment with cigarette smoke extract (CSE) $(0,1,2.5$, and $5 \%) . N=7$ experiments (2 healthy, 5 COPD donors). $n>150$ organoids/group. c Schematic of in vitro murine experimental design. d Representative images of murine lung organoids. Left: light microscopy. Right: immunofluorescence of organoids. Green: proSPC (SPC), red: acetylated- $\alpha$ tubulin (ACT), blue: DAPI. White arrow: airway type of organoid, yellow arrow: alveolar type of organoid. Scale bar, $100 \mu \mathrm{m}$. e Quantification of the normalized number of total 
concentrations of $\operatorname{CSE}(0,1.25,2.5$, and $5 \%)$. f Quantification of normalized $\mathrm{ACT}^{+}$and pro-SPC ${ }^{+}$ organoids obtained after treatment with $0,1.25,2.5$, and 5\% CSE. g Quantification of average organoid

140 diameter after treatment with $0,1.25,2.5$, and $5 \%$ CSE measured on day $14 . \mathrm{N}=5$ experiments, $n>$ 380 organoids/group. $\mathbf{h}-\mathbf{i}$ Overview of drug screening using the in vitro murine lung organoid model.

142 Comparison of the normalized number of airway and alveolar type organoids treated with the different drugs of interest in the absence (h) or presence (i) of 5\% CSE. Red bar: PTGIR, yellow bar: PTGES2, blue: antagonists. Data are presented as mean \pm SEM in number quantification. Data are presented as scatter plots with medians in size quantification. For all panels: ${ }^{* *} p<0.05,{ }^{* *} p<0.01,{ }^{* * *} p<0.001,{ }^{* * * *} p$ $<0.0001$.

We next aimed to utilize this lung organoid model to evaluate the efficacy of existing COPD therapeutics. Increasing evidence ${ }^{10,11}$ has linked phosphodiesterase (PDE) 4 inhibition to the therapeutic management of respiratory diseases, and roflumilast has been used as an oral medication in COPD patients with a prior history of hospitalization for an acute exacerbation (GOLD 2021). This led us to explore whether the classic PDE4 inhibitor, rolipram, was able to rescue the CS-induced reduction in organoid formation by alveolar progenitors. Thus, organoids were subjected in vitro to different concentrations ( 1 and $10 \mu \mathrm{M}$ ) of rolipram in the presence and absence of $5 \%$ CSE for up to 14 days (Fig.S1a). Rolipram $(10 \mu \mathrm{M})$ alone significantly increased the total number of organoids at day 7 as well as the pro-SPC ${ }^{+}$organoids at day 14 (Fig.S1b, c), but had no beneficial effects on organoid numbers when combined with CSE exposures. Treatment with rolipram $(1 \mu \mathrm{M})$ either alone or in combination with CSE induced significantly increase alveolar organoid size (Fig.S1d). models. Intriguingly, budesonide (1-, 10-, $100 \mathrm{nM})$ in combination with CSE exposure

164 further reduced the number as well as the size of both airway and alveolar type 
165 organoids as compared to CSE exposure alone (Fig. S1f, g). In vivo exposure to 166 budesonide together with CS increased the number of airway but not alveolar 167 organoids (Fig. S1i), without affecting organoid size (Fig. S1j). Taken together, these 168 data show that in vitro exposure to cigarette smoke extract functionally represses 169 human alveolar epithelial progenitor organoid formation, resulting in reduced growth 170 and differentiation, which can be mimicked using murine alveolar epithelial progenitors.

171 Validating the limitations of current pharmacology, PDE4 inhibitors and corticosteroids

172 do not prevent or reduce the detrimental effects of cigarette smoke on organoid 173 formation.

174 The assay was used subsequently to screen for the functionality of the targets in 175 restoring organoid growth. Genes downregulated in response to CS and COPD were 176 targeted with activating ligands, whereas genes upregulated in response to CS and

177 COPD were targeted using antagonists with exception of ACSS2 and CHRM3 for 178 which we included both an agonist and an antagonist. The effects of the drugs 179 targeting the 15 selected genes alone (compared to vehicle, Fig. $2 \mathrm{~h}$ ) and in the 180 presence of 5\% CSE exposure (Fig. 2i) on the number of organoids were determined. 181 Specific information of all drug effects on organoid number and size are summarized 182 in Supplementary figure 2 and 3. Interestingly, the compound activating ACSS2 183 (Acetyl-CoA synthetase short-chain family member 2), increased the number of airway 184 type organoids and the size of alveolar organoids in combination with CSE (Fig. S2185 3), whereas the ACSS2 inhibitor had the opposite effects. Atropine (CHRM3 antagonist), IBSP (Integrin Binding Sialoprotien) and LEPR (Leptin receptor) tended

187 to increase the number and size of alveolar organoids in response to CSE as well (Fig.

188 S2-3). However, considering the overall magnitude of alveolar type organoids 189 particularly, PGE2 (target gene PTGES) and iloprost, (PGI2 analogue, target gene 
190 PTGIR) were identified as the by far most promising targets with regards to their

191 capacity in restoring the CSE-induced repression of organoid formation (Fig. 2h-i, S2-

$1923)$.

193 PGE2 and PGI2 significantly prevent alveolar epithelial dysfunction. The

194 PTGES2 and PTGIR genes encode membrane-associated prostaglandin E synthase and the prostacyclin (PGI2) receptor, respectively. PGE2 acts on 4 receptor subtypes, being PTGER1-4, whereas PGI2 acts primarily on PTGIR. We assessed their expression in human lung tissue of healthy smokers and COPD patients and found maintained expression of all receptors in disease with some small differences in

199 expression, most notably a reduced expression of PTGER2 and increased expression of PTGIR (Fig. 3a). Single cell RNAseq (sc-RNAseq) data from human lung tissue shows similar expression of all 5 receptors was detected in alveolar epithelial cells and in fibroblasts (http://www.copdcellatlas.com/). Single cell RNA sequencing of mouse lung tissue showing that expression of Ptger2 and Ptger4 were highest compared to

204 that of Ptger1 and Ptger3 in mesenchymal cells (Fig. 3b, c). Interestingly, the expression of PTGES, and PTGES2, the enzymes responsible for PGE2 synthesis were relatively ubiquitous in human and mouse lung tissue, whereas PTGIS, the enzyme responsible for PGI2 synthesis was highest in mesenchymal cell types including fibroblasts. The expression of all these receptors showed lower copy numbers, which is expected for G protein-coupled receptors (GPCRs). 

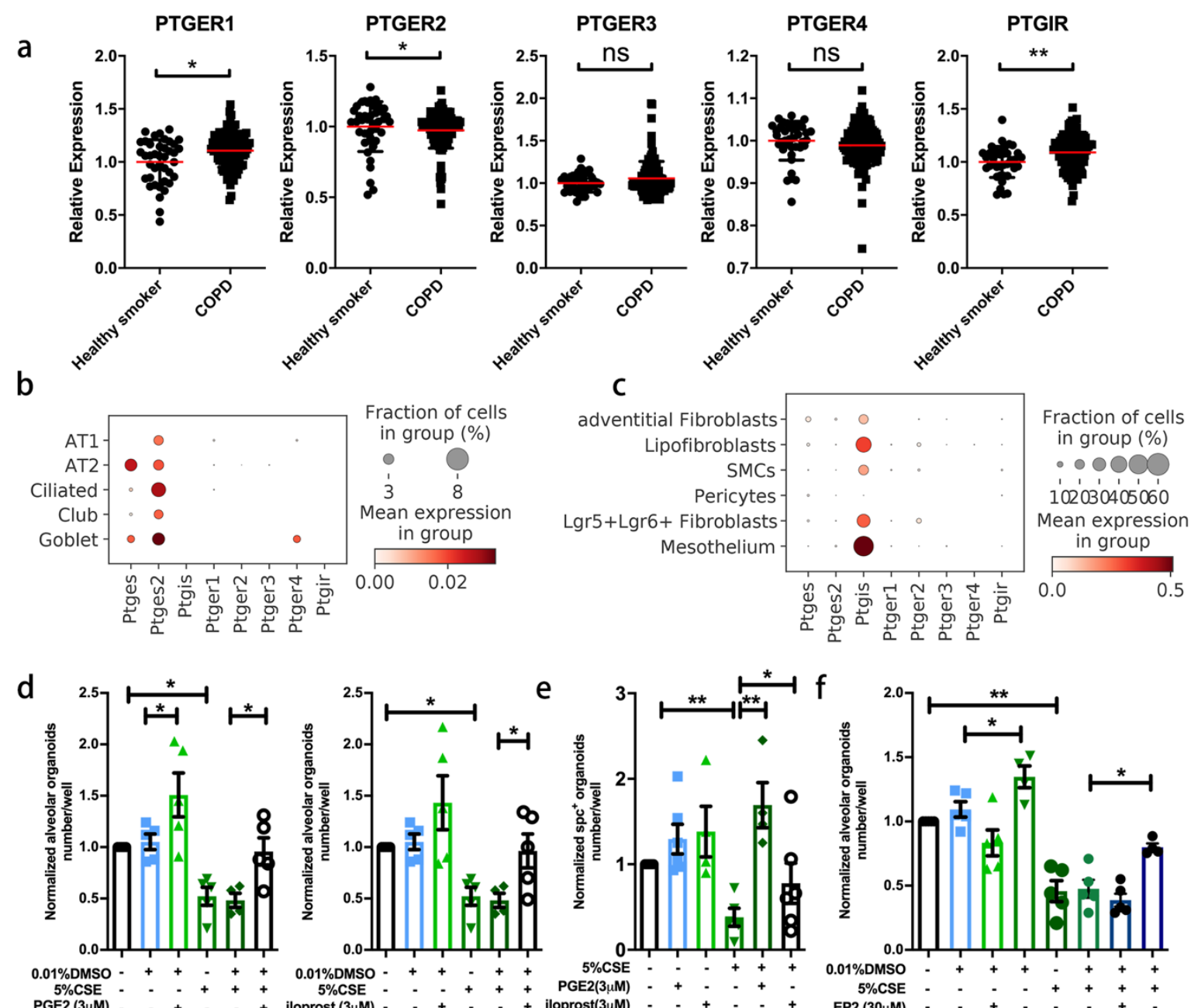

\% DMSO prost $(3 u \mathrm{M})$

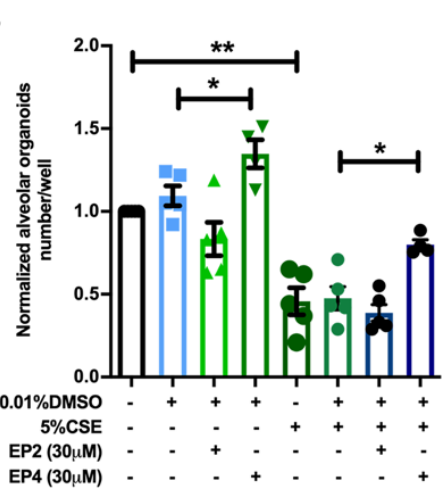

211 Figure 3 16,16-dimethyl prostaglandin E2, iloprost, and selective EP2 and EP4 analogues restore

212 lung organoid formation in response to cigarette smoke (extract). a The relative gene expression

213 of PTGER1, PTGER2, PTGER3, PTGER4 and PTGIR in healthy smokers $(\mathrm{N}=40)$ and COPD patients

$214(\mathrm{~N}=111)$ downloaded from the NCBI GEO database GSE76925. $\mathbf{b}-\mathrm{c}$ Data are extracted from the NCBI

215 GEO database GSE151674 b The expression of Ptges, Ptges2, Ptgis, Ptger1, Ptger2, Ptger3, Ptger4

216 and Ptgir in epithelial cells using scRNA-seq analysis of mouse lung tissue. c The expression of Ptges,

217 Ptges2, Ptgis, Ptger1, Ptger2, Ptger3, Ptger4 and Ptgir in mesenchymal cells using scRNA-seq analysis of mouse lung tissue. d Quantification of normalized number of alveolar type organoids treated with

219 vehicle control, or 5\% CSE \pm PGE2 agonist (16,16-dimethyl prostaglandin E2)/iloprost. e Quantification of normalized number of $\mathrm{SPC}^{+}$organoids treated with vehicle control, or $5 \%$ CSE \pm PGE2 agonist

221 (16,16-dimethyl prostaglandin E2) or iloprost. $\mathbf{f}$ Quantification of normalized number of alveolar type of 222 organoids treated with vehicle control, or 5\% CSE \pm selective EP2 or EP4 agonist. Data are presented as mean \pm SEM. ${ }^{*} p<0.05,{ }^{* *} p<0.01,{ }^{* * *} p<0.001,{ }^{* * * *} p<0.0001$. 
To further characterize the effects of PGE2 and PGI2 on defective alveolar epithelial progenitors, we examined them in vitro (Fig. 3d-f) and in vivo (Fig. 4) CS(E). The PGE2 increased the number of alveolar type organoids even in the presence of $5 \%$ CSE (Fig.

3d) and significantly increased the number of SPC ${ }^{+}$organoids under conditions of CSE exposure (Fig. 3e). To address the relative roles of the two Gs-coupled PGE2 receptors, EP2 and EP4, we evaluated the selective agonists ((R)-Butaprost and 5[(3S)-3-hydroxy-4-phenyl-1-buten-1-yl]1-[6-(2H-tetrazol-5R-yl)hexyl]-2-pyrrolidinone, CAY10598) of these receptors. Focus was on these Gs coupled receptors, as we found that cholera toxin, a well-known inducer of constitutive adenylyl cyclase activity and cAMP signaling, increased organoid formation both with and without the exposure to CSE (Fig. S4a). The EP2 selective butaprost had no effects on organoid number (Fig. 3f) but increased the alveolar size in the absence and presence of $5 \%$ CSE (Fig. S5c). The EP4 selective agonist increased the number of alveolar type organoids significantly and prevented the number reduction resulting from $5 \%$ CSE exposure (Fig. 3f). The EP4 agonist also increased the size of both types of organoids in the absence and presence of 5\% CSE (Fig. S5c). To explore whether duration of drug exposure affected organoid formation, the organoids were treated in vitro with PGE2 analogue or PGI2 analogue for 3 different time windows during organoid development as illustrated in Supplementary figure $5 \mathrm{~d}-\mathrm{g}$. These time windows were identified 244 previously $^{9}$, and mark the initial division phase (day 0-2), proliferation (day 2-7), and differentiation phase (day 7-14). We observed no effects on the number of organoids

246 for any of the short-term drug treatments, suggesting continuous treatment with or

247 iloprost during all phases of organoid formation is required. 
248 To examine the effects of PGE2 and PGI2 in vivo, we exposed mice to air (vehicle

249 control), CS, CS + misoprostol (PGE2 analogue), or CS + iloprost as shown in Figure

250 4. To assess the impact of in vivo cigarette smoke (CS) exposure, we isolated CD31-

251 /CD45-/EpCAM ${ }^{+}$cells from mice exposed to air or CS and co-cultured these with

252 CCL206 fibroblasts in vitro for 14 days (Fig. 4a). Interestingly, the number of alveolar

253 organoids was significantly decreased after in vivo CS exposure indicating that a

254 relatively short exposure to cigarette smoke in vivo is sufficient to capture early

255 changes in progenitor cell function (Fig. 4c). Cigarette smoke exposure did not change

256 the yield of CD31-/CD45-/EpCAM ${ }^{+}$cells, whereas exposing mice to misoprostol or

257 iloprost increased the yield of EpCAM+ cells (Fig. S6a). The organoid assay revealed

258 that in vivo (i.p.) treatment with either misoprostol or iloprost significantly increased the

259 number of alveolar type and $\mathrm{SPC}^{+}$organoids (Fig. 4e-f) ex vivo. Next, to investigate

260 whether in vitro drug treatment would have similar effects on damage caused by in

261 vivo CS exposure, we isolated $\mathrm{EpCAM}^{+}$cells from either air- or CS-exposed mice and

262 subjected these to in vitro misoprostol or iloprost treatment in the organoid assay for

26314 days (Fig. 4g). In vitro misoprostol increased the number of alveolar type organoids

264 in cultures derived from CS-exposed mice (Fig. 4h). Only in vitro misoprostol increased

265 the size of alveolar organoids derived from CS-exposed animals (Fig. S6c). Taken

266 together, our data show that PGE2 and PGI2 analogues protect alveolar epithelial

267 progenitor function from the effects of CS exposure. In addition, EP4 rather than EP2

268 seems to mediate the protective effects of PGE2. 
a

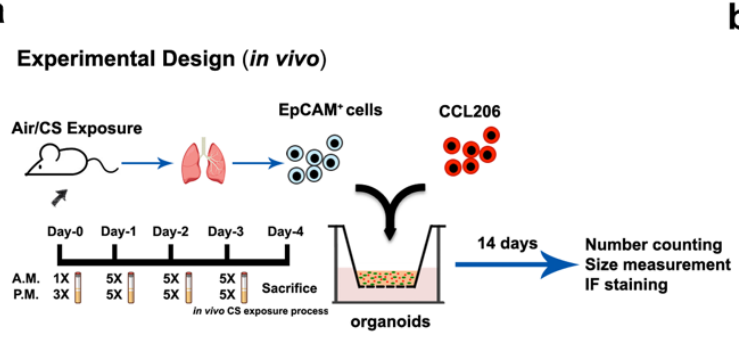

d

Experimental Design (in vivo)
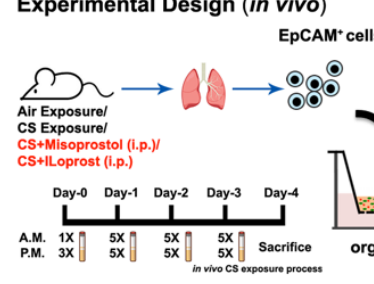

:.M.

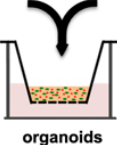

14 days Number counting

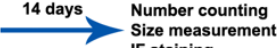

organoids

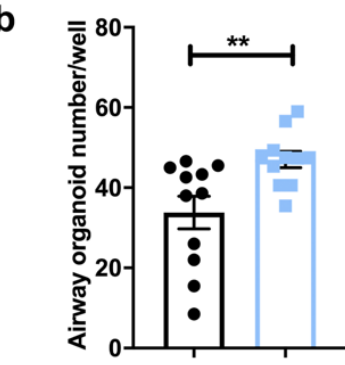

e

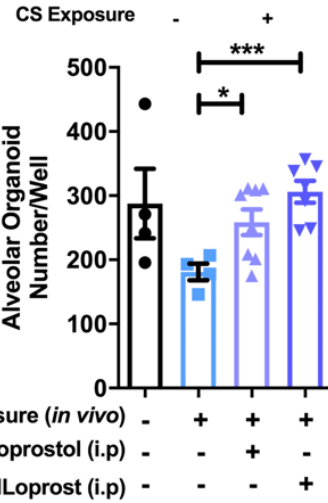

g

Experimental Design (in vivo)
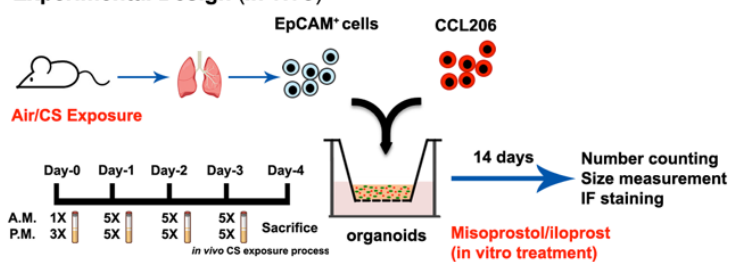
Misoprostol/iloprost
(in vitro treatment)
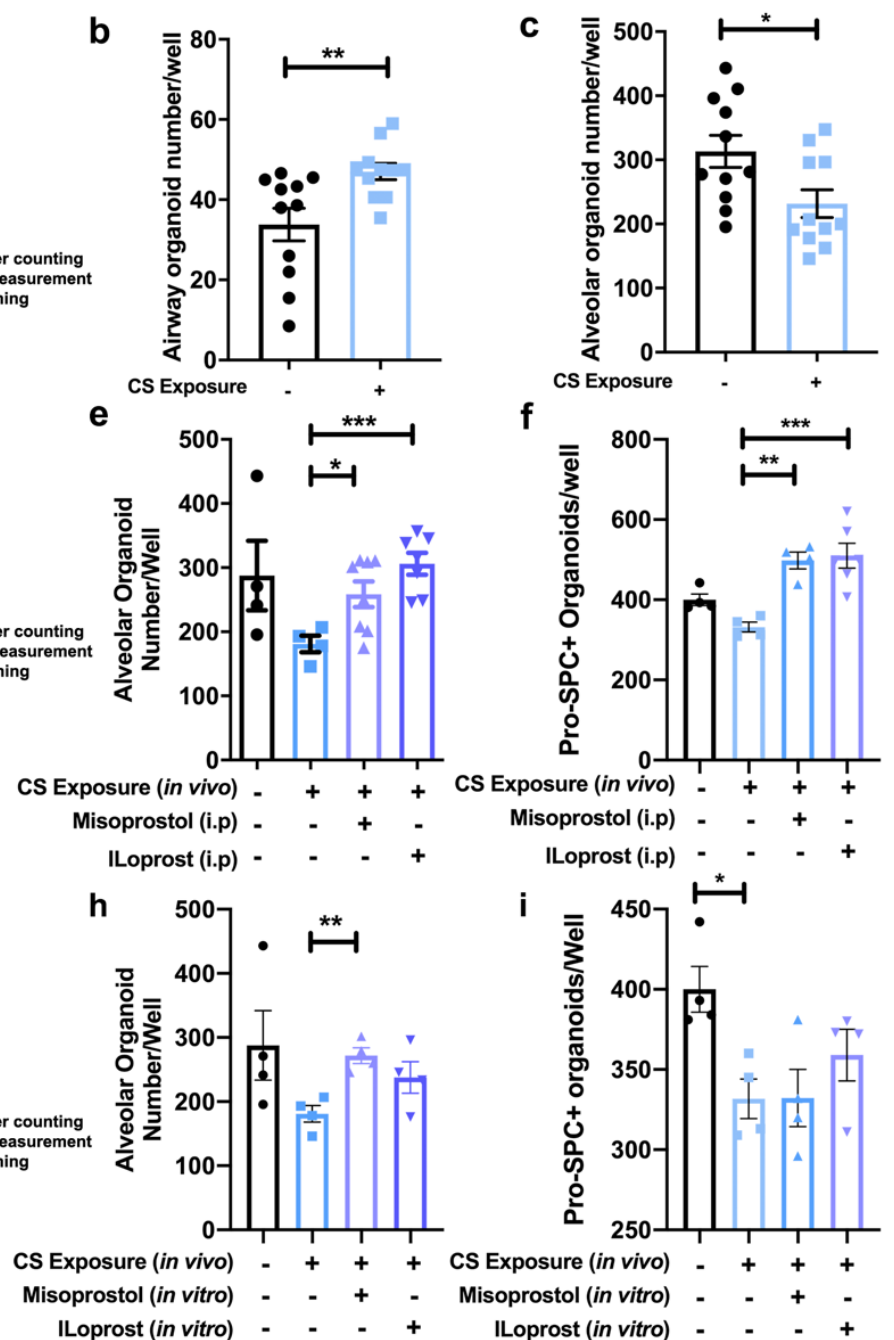

f

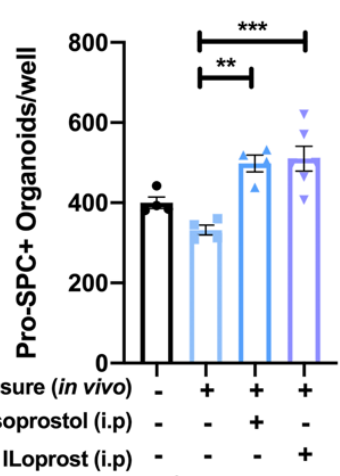

i

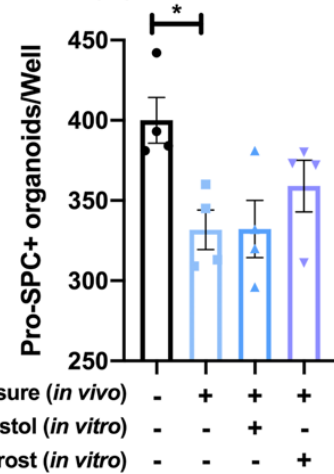

Figure 4 Administration (in vivo and in vitro) of misoprostol and iloprost to cigarette smoke-

exposed mice restored lung organoid formation. a Schematic of in vivo CS exposure experimental setup. b-c Number of airway and alveolar type organoids quantified on day 14 of co-culturing CCL-206 fibroblasts and $\mathrm{EpCAM}^{+}$cells (isolated from air exposed/CS exposed mice). $\mathrm{N}=11$ experiments. $\mathbf{d}$ Schematic of experimental design. Organoids were generated from air- or CS-exposed mice treated in vivo with misoprostol (i.p.) or iloprost (i.p.); all organoids were treated with normal organoid medium. ef Number of alveolar type and pro-SPC ${ }^{+}$organoids quantified on day 14 from co-culture of CCL-206 fibroblasts and $\mathrm{EpCAM}^{+}$cells (isolated from air- (control) and CS-exposed mice treated intraperitoneally with misoprostol or iloprost). $\mathbf{g}$ Schematic of experimental design. Organoids were generated from mice exposed to air or CS. Misoprostol and iloprost were added in vitro to the organoid medium for treatment. h-i Number of alveolar type and SPC+ organoids quantified on day 14 from co-culture of CCL-206 fibroblasts and $\mathrm{EpCAM}^{+}$cells (isolated from air- and CS-exposed mice) treated with misoprostol/iloprost in vitro. Data are presented as mean \pm SEM. ${ }^{*} p<0.05,{ }^{* *} p<0.01,{ }^{* * *} p<0.001,{ }^{* * * *} p<0.0001$. 
283 Distinct genetic signatures in regulation of defective alveolar epithelial repair.

284 To unravel the transcriptional changes leading to impaired lung organoid formation

285 after exposure to CS as well as the mechanisms underlying the beneficial effects of

286 PGE2 and PGI2 treatment, we performed RNA sequencing (Fig. 5a) on EpCAM+ cells

287 isolated from mice exposed to air (control), CS, CS + misoprostol(i.p.), or CS + iloprost

288 (i.p.), directly after the isolation procedure (i.e., prior to inclusion in the organoid assay).

289 Principal-component analysis (PCA) revealed that the CS-exposed group is

290 transcriptionally distinct from the control group (Fig. 5b), and that the CS/misoprostol

291 and CS/iloprost groups are transcriptionally different from the CS exposed group. The

292 top 20 differentially expressed genes from these three comparisons, including both

293 up- and downregulated genes, are shown in the heatmaps (Fig. 5c) and summarized

294 in supplementary materials. Reactome pathway analysis was used to identify

295 molecular pathways overrepresented in CS/misoprostol/iloprost-modulated genes in

296 alveolar epithelial cells. Within the top 20 enriched pathways (Fig. 5d, supplementary

297 materials), genes associated with cell cycle, mitotic prometaphase, DNA

298 replication/synthesis and RHO GTPases activate formins signaling pathways were

299 upregulated by CS exposure compared to air (control) exposure, however, these were

300 downregulated by treatment with misoprostol or iloprost. Notably, genes associated

301 with the circadian clock signaling pathway were downregulated by CS exposure but

302 restored by treatment with misoprostol or iloprost. Moreover, signaling by FGFR1, 3

303 and 4 were downregulated in response to CS exposure, whereas the same signaling

304 pathways were upregulated by misoprostol but not iloprost treatment. These findings

305 suggest that the repairing mechanisms of misoprostol and iloprost in response to CS

306 were common in cell cycle and circadian clock signaling and the distorted FGFR

307 signaling resulted from CS was corrected by misoprostol uniquely. In addition, the 
309 WNT and cell-cell communication signaling pathways appear to downregulate in

310 response to CS, which were not observed in either misoprostol/iloprost treatment

311 group. Overall, the RNA-seq analysis demonstrates both common and distinct

312 transcriptomic mechanisms of misoprostol and iloprost treatment in response to CS

313 exposure in alveolar epithelial progenitors.

a
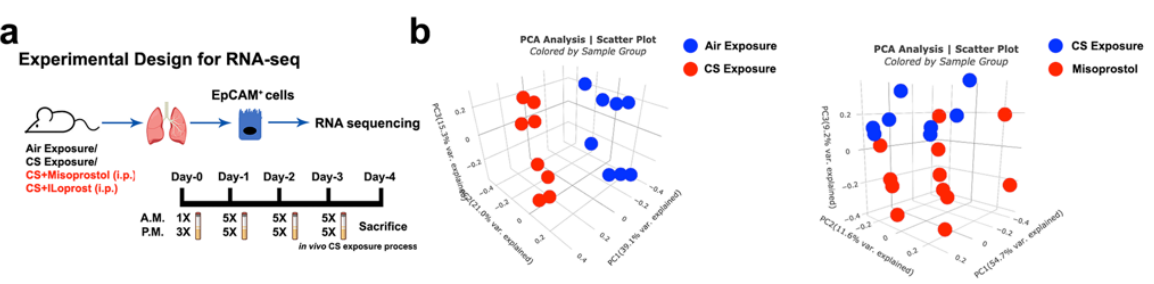

C
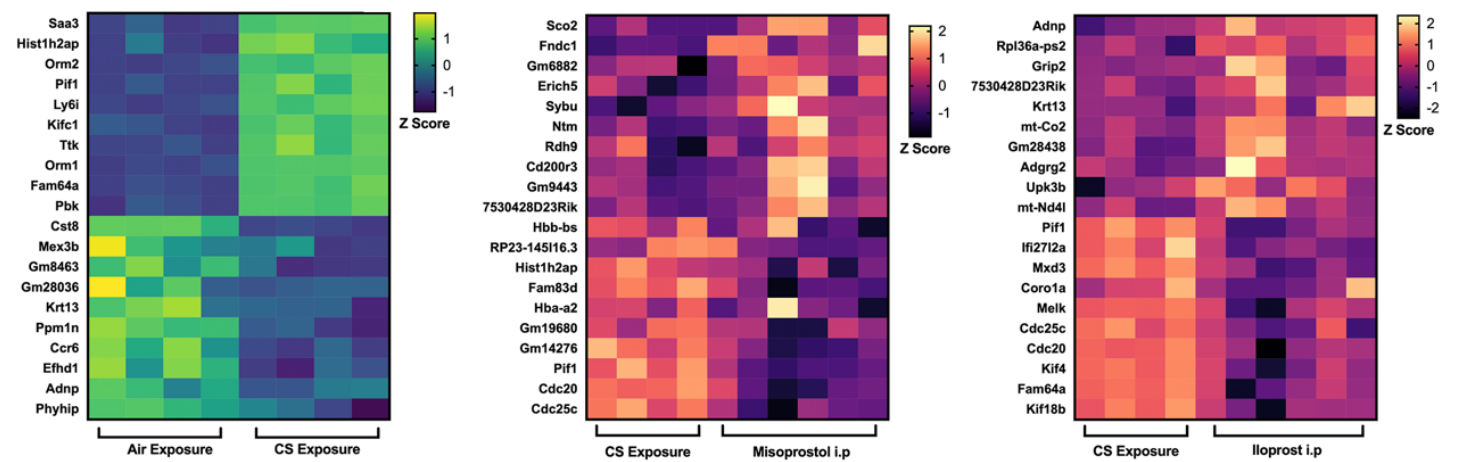

d

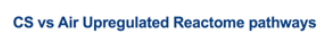

Misoprostol vs CS Upregulated Reactome Pathways
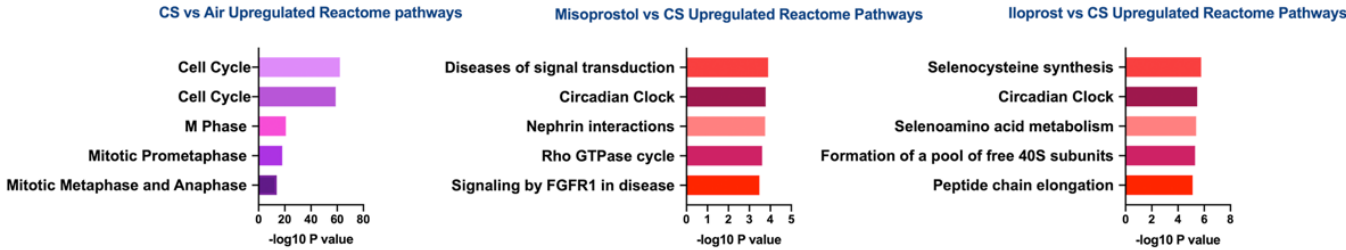

CS vs Air Downregulated Reactome pathways
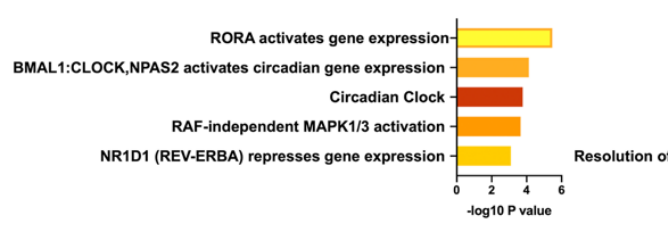
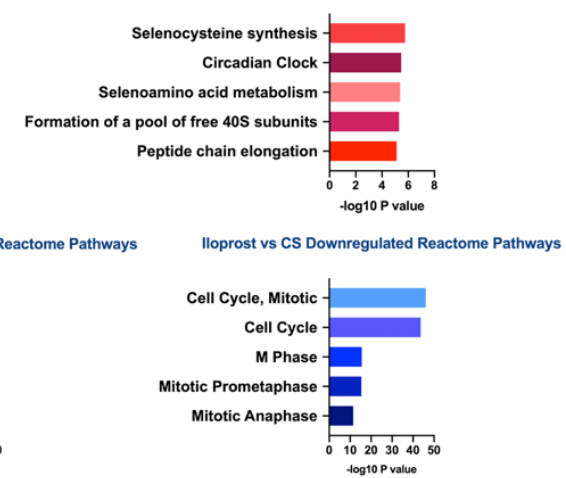

Figure 5 Transcriptomic signatures in response to cigarette smoke with(out) misoprostol and

316 iloprost. a Schematic experimental design for RNA sequencing. b PCA plots demonstrate the clusters

317 betweern different comparisons: air vs CS, CS vs CS+misoprostol, and CS vs CS+iloprost. c Heatmaps

318 displaying the top 20 differentially expressed genes in air vs CS, CS vs CS+misoprostol, and

319 CS+iloprost exposed epithelial cells. d The top 5 significantly up- and downregulated reactome 
pathways enrichment form differentially expressed genes within the comparisons of air vs CS exposure,

CS exposure vs CS+misoprostol, and CS exposure vs CS+iloprost.

\section{Discussion}

Chronic obstructive pulmonary disease results from repeated micro-injuries to the epithelium often caused by tobacco smoking. In susceptible individuals, this results in tissue remodeling in the conducting airways and destruction of the respiratory bronchioles and alveoli ${ }^{19}$. No clinically approved pharmacological treatment prevents or reverses the tissue destruction in the distal lung. The results of our study are in line with this contention and demonstrate that the PDE4 inhibitor rolipram and the

330 corticosteroid budesonide had no, or only very limited, beneficial effects on impaired organoid growth and differentiation in response to cigarette smoke extract in vitro or in response to cigarette smoke exposure in vivo. In fact, if anything, budesonide appeared to restrict progenitor cell growth, which is a concern given the wide use of corticosteroids in the management of COPD. This underscores the need for novel drug targets.

336 Consequently, we set out to search for new potential drug targets for lung repair in

337 COPD and identify EP and IP receptor agonists as two such potential targets, by 338 utilizing a transcriptomics-guided drug discovery strategy. Both EP and IP receptor 339 agonists were able to promote epithelial repair responses after exposure to cigarette 340 smoke (extract). Whereas PGE2 and PGI2 showed the most profound changes, other 341 methods including ACSS2 agonism, LEPR agonism and IBSP agonism yielded smaller effects. ACSS2 supports acetyl-CoA synthesis from acetate in the cytosol ${ }^{20,21}$,

343 and thereby plays an important role in lipid metabolism and in the regulation of histone

344 acetylation in the nucleus during gene transcription. IBSP is a member of the small 
345 integrin-binding ligand N-liked glycoprotein (SIBLING) family ${ }^{22,23}$, which is associated

346 with bone metastases of lung cancer ${ }^{24}$. LEPR (leptin receptor), is an adipocytokine

347 that has a central role in regulating food intake and energy expenditure ${ }^{25}$, but has also

348 been linked to lung function decline in a population in COPD ${ }^{26}$. Nonetheless, among

349 all the candidate targets, PGE2 (PTGES2) and PGI2 (PTGIR) analogues emerged as

350 the most promising compounds among all drugs in the current study. Prostaglandins

351 (PGs) are lipid mediators synthesized from arachidonic acid (AA) via the cyclooxygenase pathway, and include PGD2, PGI2, PGH2, PGE2 and PGF2 $\alpha^{27}$. PGI2 signals via IP receptors to induce cAMP signaling, similar to EP4 receptors. We show EP4 receptors to have similar expression as in non-COPD controls, whereas IP receptors are expressed at higher levels in COPD patients indicating the expression of both receptors is maintained in disease.

We show that PGE2 agonists are beneficial in reducing CS-induced damage in alveolar epithelial progenitors. However, PGE2 has been reported as an unstable molecule with an extremely short half-life; therefore, targeting its receptors with specific more stable analogues may be a better alternative. PGE2 is the most widely produced PG in the human body and it signals via four specific G-protein-coupled receptors $\left(\mathrm{EP} 1-4^{28,29}\right)$. The interactions between PGE2 and EP receptors depend on tissue and cell type, specific receptor expression, and differences in binding affinities,

364 leading to unique patterns of EP receptor activation ${ }^{30}$. PGE2 can stimulate cAMP 365 production through EP2 and EP4 receptors, whereas EP3 activation results in decreased cAMP synthesis and EP1 stimulation is coupled to Gq-activation and

367 (enhanced) $\mathrm{Ca}^{2+}$ signaling ${ }^{27,30,31}$. EP1 and EP3 receptors can mediate

368 bronchoconstriction indirectly through activation of neural pathways ${ }^{32}$, as a 369 consequence non-selective PGE2 analogues are unsuitable as pulmonary drugs. 
370 Therefore, we selected analogues of EP2 and EP4 to mimic effect of PGE2 in our

371 organoid assay, and demonstrated that EP4 agonism showed beneficial effects

372 against impaired organoid formation in response to (CS)E exposure. Targeting EP4

373 receptors is worthwhile investigating in more detail in the future, as the effects may

374 surpass epithelial repair only. Additional beneficial effects of EP4 agonism in COPD

375 may include bronchoprotection ${ }^{33}$ and inhibition of inflammation ${ }^{34}$, suggesting that EP4

376 agonism could unify several functional features that support the treatment of COPD,

377 making this an intriguing pharmacological target.

378 Iloprost, a stable PGI2 analogue ${ }^{27,35-39}$, has been shown to have anti-inflammatory

379 effects and protects against bleomycin-induced pulmonary fibrosis in mice ${ }^{36}$, and is

380 also clinically used for the treatment of pulmonary hypertension ${ }^{35}$. Although a recent

381 study ${ }^{38}$ showed iloprost improved clinical outcomes in COPD patients with poor lung

382 oxygenation, its impact on alveolar repair is unknown. Here we show that iloprost

383 prevents the repressed organoid formation resulting from CS(E) exposure.

384 By generating transcriptomic signatures of epithelial progenitors derived from mice

385 exposed in vivo to air, CS, CS + misoprostol or CS + iloprost, we uncovered dynamic

386 molecular signaling pathways in response to CS exposure. Intriguingly, we identified

387 circadian clock signaling as being significantly repressed in the alveolar epithelial

388 progenitors derived from mice exposed to CS, which could be improved by either

389 misoprostol or iloprost treatment. Circadian rhythms ${ }^{40-43}$ are normally generated and

390 regulated by clock genes, including BMAL1 (ARNTL1) and CLOCK encoding

391 activators, period (PER1-3) and cryptochrome genes (CRY1-2) that encode

392 repressors, and the nuclear receptors Rev-erb (NR1D1 and NR1D2) and RORA which

393 constitute secondary regulatory loops. These core clock genes not only activate or

394 repress a cell-autonomous clock, but also regulate the clock-controlled genes 
$395(\mathrm{CCGs})^{44}$, thus interacting with other molecular signaling pathways. Previously, it has

396 been demonstrated that clock signaling is downregulated in CS exposed mice, linked

397 to an impairment of anti-oxidant defense mechanisms ${ }^{45}$ and Rev-erba has been

398 shown as an key regulator of inflammatory response in lung injury models ${ }^{16-18,46}$

399 Furthermore, we found that CS exposure upregulated pathways associated with cell

400 cycle activity in alveolar epithelial progenitors, which could be counteracted by in vivo

401 misoprostol or iloprost treatment. The cell cycle ${ }^{47-51}$ is driven by a set of tightly

402 regulated molecular events controlling DNA replication and mitosis with four phases,

403 and each individual cell may require different triggers in order to decide whether to

404 enter proliferation or apoptosis. To further assess alveolar epithelial progenitors under

405 which cell cycle/apoptotic status in response to CS exposure as well as additional

406 PGE2/PGI2 treatments may be the next step to investigate in the future. A link between

407 circadian clock and cell cycle signaling pathways has been proposed $44,50,52$.

408 Importantly, the molecular control of the biological clock is dependent on cAMP

409 signaling and cAMP activators are known to entrain the biological clock $^{53}$, explaining

410 the link between PGE2 and PGI2 activation and restoration of the defective clock

411 signaling in combination with CS exposure. Hence, it is of great interest to determine

412 in more molecular detail how these two oscillatory systems communicate in regulating

413 PGE2/PGI2-mediated lung repair in future studies.

414 In conclusion, in this study we demonstrate for the first time the protective effects of

415 several drug candidates, and in particular PGE2 and PGI2 analogues, against in vivo

416 and in vitro $\mathrm{CS}(\mathrm{E})$-induced damage of alveolar epithelial progenitors. Furthermore,

417 using transcriptome analysis, we show that CS induces a wide range of transcriptional

418 changes, including alterations of circadian clock and cell cycle signaling pathways,

419 which can be counteracted by either misoprostol (PGE2) or iloprost (PGI2) treatment. 
420 Overall, these data provide promising therapeutic strategies to specifically address

421 defective lung repair in respiratory diseases, in particular targeting EP4 and IP 422 receptors. 
424 Animals. All mouse experiments for organoid study were performed at the Central 425 Animal Facility (CDP) of the University Medical Center Groningen (UMCG) in accordance with the national guidelines and upon approval of the experimental procedures by CDP and the Institutional Animal Care and Use Committee (IACUC) of the University of Groningen (CCD license AVD105002015303). C57BL/6J (555) and BALB/cByJ (Jax-strain) mice (both genders, 8-12 weeks of age) were maintained under 12-h light/ dark cycles and were allowed food and water ad libitum. Animals for circadian clock studies were exposed to CS and/or administrated with compounds at the same time of the day for all mice in all groups. Animals were euthanized at the same time of the day. Adult (female, 8-10 weeks of age) C57BL/6N mice were obtained from Charles River (Sulzfeld, Germany) for the single cell RNAseq analysis of lungs following exposure to chronic CS. These experiments were performed at the Helmholtz Zentrum München and approved by the ethics committee for animal welfare of the local government for the administrative region of Upper Bavaria (Regierungspräsidium Oberbayern) and were conducted under strict governmental and international guidelines in accordance with EU Directive 2010/63/EU.

Human material. The human lung tissue was obtained from lung transplant donors according to the Eurotransplant guidelines including the absence of primary lung diseases such as asthma and COPD, and no more than 20 pack years of smoking 444 history ${ }^{54}$. Gene expression in human lung published data sets was obtained by down 445 loading series matrix files from the NCBI GEO database for GSE76925 55 and gene expression normalized to healthy smokers. 
448 In vivo cigarette smoke exposure. Mice ( $\mathrm{n}=4-11 /$ group, $10-12$ weeks old) were 449 exposed (whole body) to 3R4F research cigarettes (Tobacco Research Institute, 450 University of Kentucky, Lexington, KY) for four consecutive days (two sessions/day, 8 451 hours between each exposure) to establish an acute smoke-induced inflammation 452 model, as described previously ${ }^{8}$. In the cigarette smoke (CS) group, mice were 453 exposed to 1 cigarette in the morning and 3 in the afternoon on day 1 . From day 2 to 4544 , mice were exposed to 5 cigarettes each session. All cigarettes were smoked without a filter in $5 \mathrm{~min}$ at a rate of $5 \mathrm{~L} / \mathrm{h}$ in a ratio with $60 \mathrm{~L} / \mathrm{h}$ air using a peristaltic pump (45 456 rpm, Watson Marlow 323 E/D, Rotterdam, NL). In the control group, mice were exposed to fresh air using similar exposure chambers as the CS group.

In some studies, budesonide was nebulized $(0.1 \mathrm{mM}, 15 \mathrm{~min} / \mathrm{mouse} / \mathrm{exposure})$ to wild-type mice $(n=6)$ prior to each CS exposure. In separate studies, intraperitoneal (IP) injections of $50 \mu \mathrm{g}$ misoprostol or $50 \mu \mathrm{g}$ iloprost were given 30 min to wild-type 461 mice $(n=6-8)$ prior to each CS exposure. On day 5 , mice were sacrificed, and the 462 lungs were immediately used for establishing organoid cultures or stored at $-80^{\circ} \mathrm{C}$ for 463 further experimental uses.

464 For the single cell RNAseq analysis of lungs CS was generated from 3R4F 465 Research Cigarettes with the filters removed. Mice were whole body exposed to active $466100 \%$ mainstream CS of $500 \mathrm{mg} / \mathrm{m}^{3}$ total particulate matter (TPM) for 50 min twice per 467 day for $4 \mathrm{~m}$ in a manner mimicking natural human smoking habits as previously 468 described $^{55}$

470 Fibroblast culture. Mouse fibroblasts, CCL206 (Mlg [CCL206], ATCC, Wesel, 471 Germany) were cultured in DMEM/F12 medium supplemented with $10 \%$ (v/v) fetal 472 bovine serum, $100 \mathrm{U} / \mathrm{mL}$ penicillin/streptomycin, $2 \mathrm{mM}$ L-glutamine, and 1\% 
473 amphotericin $\mathrm{B}$ in a humidified atmosphere under $5 \% \quad \mathrm{CO}_{2} / 95 \%$ air at $37^{\circ} \mathrm{C}$, as

474 previously described ${ }^{6,9,56}$. For organoid experiments, fibroblasts were proliferation-

475 inactivated by incubation in mitomycin C (10 $\mathrm{gg} / \mathrm{mL}$, Sigma, M4287) for $2 \mathrm{~h}$, followed

476 by 3 washes with PBS after which the cells were trypsinized before introduction into

477 the organoid co-cultures. Human lung fibroblasts MRC5 (CCL-171; ATCC, Wesel,

478 Germany) were cultured in Ham's F12 medium supplemented with the same additives 479 as the murine fibroblasts medium.

480

481 Cigarette smoke extract (CSE). The smoke of two 3R4F research cigarettes was 482 pumped into $25 \mathrm{~mL}$ warm fibroblasts culture medium to produce $100 \%$ cigarette smoke 483 extract (CSE) ${ }^{11}$. All cigarettes were without a filter and smoke was passed through the 484 medium using a peristaltic pump (45 rpm, Watson Marlow $323 \mathrm{E} / \mathrm{D}$, Rotterdam, NL). CSE was freshly prepared before each set of experiments.

487 Organoid culture. The organoid culture system is based on previously published 488 protocols from our group ${ }^{6,9,56}$. In brief, epithelial cells (CD31/CD45/CD326 ${ }^{+}$) were freshly isolated from murine or human lung tissue and co-cultured with murine CCL206 or human MRC5 fibroblasts, respectively, in Matrigel ${ }^{\circledR}$ (Corning Life Sciences B.V.,

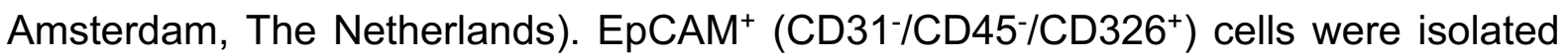

492 from mouse lung tissue (without the trachea) using the QuadroMACS TM Separator and 493 antibody-bound magnetic beads (Miltenyi Biotec, Leiden, The Netherlands). EpCAM+ 494 cells and fibroblasts were mixed $1: 1(20,000$ cells each) and suspended in $100 \mu \mathrm{L}$ of Matrigel prediluted 1:1 (v/v) with DMEM supplemented with 10\% FBS. This mixture of cells was added to a 24-well Falcon $®$ cell culture insert (Corning, USA) within a 24well plate containing $400 \mu \mathrm{L}$ of organoid media (DMEM/F-12 supplemented with $5 \%$ 
498 FBS, 1\% penicillin/streptomycin, 1\% glutamine, 1\% amphotericin B, 0.025\% EGF, $1 \%$

499 insulin-transferrin-selenium, and $1.75 \%$ bovine pituitary extract) underneath the insert

500 in each well. Adult human donor tissue was isolated from histologically normal regions

501 of lung tissue specimens obtained at University Medical Centre Groningen (Groningen,

502 The Netherlands) from $n=7$ patients (2 non-COPD and 5 COPD patients). Human

503 lung tissues were incubated and homogenized overnight in an enzyme mixture at $4{ }^{\circ} \mathrm{C}$;

504 the $\mathrm{EpCAM}^{+}$isolation process was similar to that described above for murine lung

505 tissue. Organoids were cultured in a humidified atmosphere under $5 \% \mathrm{CO}_{2} / 95 \%$ air at

$50637^{\circ} \mathrm{C}$ and medium in the wells was refreshed every 2-3 days. To quantify the number

507 of organoids, light microscopy at 20x magnification was used and organoids were

508 counted manually. The diameter of the organoids (organoid size) was measured using

509 NIS-Elements software with a light microscope.

510 For in vitro organoid experiments, organoids were continuously treated with control,

$511 \quad 1.25 \%$ ( $1 \%$ for human organoids), $2.5 \%$ or $5 \%$ CSE and organoid culture medium was

512 refreshed every other day. All information on the pharmacological compounds used in

513 this study is provided in the Supplementary table 1.

515 Immunofluorescence staining. The immunofluorescence staining assay for

516 organoids was performed as described previously by our group with minor

517 modifications ${ }^{6,9,56}$. Organoids were fixed in acetone diluted 1:1 (v/v) with methanol for

$51815 \mathrm{~min}$ at $-20{ }^{\circ} \mathrm{C}$. After fixation, one $\mathrm{mL}$ of PBS with $0.02 \%$ sodium azide was added

519 to the well underneath the insert. Organoids were kept at $4^{\circ} \mathrm{C}$ for one week after

520 fixation. BSA media was added on top of the insert for blocking at room temperature

521 (RT) for $2 \mathrm{~h}$. Afterwards, primary antibody incubation was performed in PBS buffer with

$522 \quad 0.1 \%$ BSA and $0.1 \%$ Triton $\mathrm{X}-100$ overnight at $4{ }^{\circ} \mathrm{C}$. The next day, the organoids were 
523 washed with PBS for 30 min three times and secondary antibody incubation was

524 performed for $2 \mathrm{~h}$ at RT. After washing with PBS for $15 \mathrm{~min}$, the organoids on the insert

525 membrane were transferred to a glass slide with two drops of mounting medium

526 containing DAPI (Abcam 104139, Cambridge, UK), and a coverslip was applied.

527 The slides were kept at $4{ }^{\circ} \mathrm{C}$. Confocal images were acquired using a Leica SP8

528 microscope or a Leica DM4000B microscope. Images were obtained and analyzed

529 with LASX (Leica) software (open resource, Leica Microsystems GmbH, Wetzlar,

530 Germany).

532 RNA extraction and RNA sequencing (RNA-seq) analysis. The EpCAM ${ }^{+}$cells

533 isolated from mice exposed to Air, CS, CS+misoprostol, or CS+iloprost were used to 534 extract total RNA for RNA sequencing using NucleoSpin ${ }^{\circledR}$ RNA kit (Machery-Nagel, 535740955 , Germany) according to the manufacturer's instructions. RNA concentrations 536 and qualities were analyzed using Nanodrop spectrophotometer. An Illumina NovaSeq

5376000 sequencer was used for RNA-seq data analysis by GenomeScan 538 (https://www.genomescan.nl). The procedure included data quality control, adapter 539 trimming, alignment of short reads and feature counting. Library preparation was 540 checked by calculating ribosomal (and globin) content. Checks for possible sample 541 and barcode contamination were performed and a set of standard quality metrics for 542 the raw data set was determined using quality control tools (FstQC v0.34 and FastQA).

543 Prior to alignment, the reads were trimmed for adapter sequences using Trimmomatic 544 v0.30. To align the reads of each sample, the ensemble mouse reference GRCm38 545 (patch 6) was used. Data analyses following the RNA-seq studies were performed 546 using the BioJupies platform (https://amp.pharm.mssm.edu/biojupies/) ${ }^{57}$. Gene 
547 expression in murine lung published data sets was obtained by downloading series

548 matrix files from the NCBI GEO database GSE151674.

549

550 Statistics analysis. All data are presented as mean \pm SEM unless indicated otherwise.

551 Unless stated otherwise, all data were assessed for statistical significance using two-

552 tailed Student's t-test or one-way ANOVA. The p-value indicating statistically 553 significant differences between the mean/median values are defined as follows: * $p<$ $5540.05,{ }^{* *} p<0.01,{ }^{* * *} p<0.001,{ }^{* * *} p<0.0001$. Statistical analyses were performed with 555 GraphPad Prism 8 software. 


\section{Acknowledgements:}

557 The authors would like to acknowledge the support from the Lung foundation

558 Netherlands (Longfonds, grant 5.1.17.166) and China Scholarship Council

559 (CSC201707720065).

560

561 Author Contributions:

562 Author X.W., R.G. conceptualized the project, analyzed and interpreted the data; R.G.,

563 L.E.M.K., and M.S. supervised the project; X.W., A.M., M.K., L.E.M.K., A.Ö.Y., and

564 R.G. assisted designing the experiments; X.W., S.B., V.V., L.A.V., and A.D'M. 565 performed the experiments; X.W., T.M.C., and M.A. prepared the figures; T.M.C., M.A., 566 H.B.S., and A.Ö.Y. assisted in bioinformatics analysis; X.W., and R.G. wrote the 567 manuscript; All authors reviewed and commented on the manuscript and agreed to 568 the final version.

570 Competing interest:

571 Author V.V. and L.E.M.K. are employees of Aquilo BV. Author R.G. and M.K. are 572 members of the BREATH consortium funded by the Lung foundation Netherlands 573 (Longfonds). All other authors declare no competing interests. 


\section{Supplementary tables and figures}

Supplementary Table 1 Information of final drug lists screened on organoid assay.

\begin{tabular}{|c|c|c|c|c|c|c|}
\hline NO & Overlap Up & Full name & Drug/compound name & $\begin{array}{l}\text { Final } \\
\text { concentrations }\end{array}$ & Article No. & Company \\
\hline 1 & CPS1 & Carbamoyl-phosphate synthase 1 & N-Acetyl-L-glutamic acid & $10 \mu \mathrm{M}$ & $855642-25 G$ & Sigma-Aldrich \\
\hline 2 & CTSC & Cathepsin C/Dipeptidyl peptidase 1 & Cathepsin c inhibitor & $1 \mu \mathrm{M}$ & BI-9740 & $\begin{array}{l}\text { Boehringer } \\
\text { Ingelheim }\end{array}$ \\
\hline 3 & CXCL1 & C-X-C Motif Chemokine Ligand 1 & Reparixin (L-Lysine salt) & $100 \mathrm{nM}$ & Cayman - 21492 & Cayman chemical \\
\hline 4 & DMKN & Derrmokine & Dermokine beta recombinant protein antigen & $100 \mathrm{ng} / \mathrm{mL}$ & NBP1-86840PEP & Novus Biologicals \\
\hline 5 & PTGIR & Prostaglandin I2 Receptor & iloprost & 0.03-, 0.3-, $3 \mu \mathrm{M}$ & SML1651 & Sigma-Aldrich \\
\hline 6 & RAB8B & RAB8B, Member RAS Oncogene Family & $\begin{array}{l}\text { CHEMBL384759/Guranosine 5'-diphosphate sodium salt } \\
\text { (GDP) }\end{array}$ & $10 \mu \mathrm{M}$ & G7127 & Sigma-Aldrich \\
\hline 7 & TNNI3K & TNNI3 Interacting Kinase & GSK-114 & $10 \mu \mathrm{M}$ & & \\
\hline NO & Overlap Down & Full name & Drug/compound name & & Article No. & Company \\
\hline 1 & ACSS2 & Acyl-CoA Synthetase Short Chain Family Member & Acetyl coenzyme A sodium salt & $10 \mathrm{mM}$ & A2056-1MG & Sigma-Aldrich \\
\hline 2 & Acss $2 \mathrm{i}$ & 2 & ACSS2 inhibitor & $10 \mathrm{mM}$ & S8588 & Selleckchem.com \\
\hline 3 & CHRM3 & Cholinergic Receptor Muscarinic 3 & Methacoline & $10 \mu \mathrm{M}$ & A2251 & Sigma-Aldrich \\
\hline 4 & CHRM3i & & Atrophine & $1 \mu \mathrm{M}$ & A0132 & Sigma-Aldrich \\
\hline 5 & FGF17 & Fibroblast Growth Factor 17 & Recombinant human FGF-17 protein & $10 \mathrm{ng} / \mathrm{mL}$ & 319-FG-025 & R\&D systems \\
\hline 6 & IBSP & Integrin Binding Sialoprotein & Recombinant Human IBSP/Sialoprotein II Protein, CF & $10 \mathrm{ng} / \mathrm{mL}$ & 4014-SP-050 & R\&D systems \\
\hline 7 & LEPR & Leptin Receptor & Recombinant Mouse Leptin Protein, CF & $100 \mathrm{ng} / \mathrm{mL}$ & L4146-1MG & Sigma-Aldrich \\
\hline \multirow[t]{2}{*}{8} & PTGES2 & Prostaglandin E Synthase 2 & 16,16-dimethyl Prostaglandin E2 & 0.03-, 0.3-, $3 \mu \mathrm{M}$ & D0160 & Sigma-Aldrich \\
\hline & & & Misoprostol & $3 \mu \mathrm{M}$ & M6807 & Sigma-Aldrich \\
\hline 9 & SLC16A3 & Solute Carrier Family 16 Member 3 & Streptozocin & $10 \mu \mathrm{M}$ & S0130-50MG & Sigma-Aldrich \\
\hline 10 & SLC1A4 & Solute Carrier Family 1 Member 4 & L-Alanine & $100 \mu \mathrm{M}$ & A7627-1G & Sigma-Aldrich \\
\hline
\end{tabular}


Supplementary Table 2 Information of compounds used in organoid assay.

\begin{tabular}{llll}
\hline Name & Final concentrations & Article No. & Company \\
Rolipram & $1-, 10 \mu \mathrm{M}$ & R6520 & Sigma-Aldrich \\
Budesonide & $1-, 10-, 100 \mathrm{nM}$ & B 7777 & Sigma-Aldrich \\
Cholera toxin & $0.1 \mathrm{mg} / \mathrm{mL}$ & $\mathrm{C} 8052$ & Sigma-Aldrich \\
(R) -Butaprost (EP2 analogue) & $30 \mu \mathrm{M}$ & $\mathrm{B} 6309$ & Cayman chemical \\
EP4 analogue* & $30 \mu \mathrm{M}$ & CAY10598 & Sigma-Aldrich \\
Forskolin & $10 \mu \mathrm{M}$ & F6886 & Obtained from AppliChem (Germany) \\
IBMX (3-Isobutyl-1-methylxanthine) & $100 \mu \mathrm{M}$ & D0627 & Sigma-Aldrich \\
db-cAMP (Bucladesine) & $100 \mu \mathrm{M}$ & Obtained from AppliChem (Germany) \\
\hline Olodaterol & $100 \mathrm{nM}$ & & \\
\hline
\end{tabular}

*EP4 analouge: 5-[(3S)-3-hydroxy-4-phenyl-1-buten-1-yl]1-[6-(2H-tetrazol-5R-yl)hexyl]-2-pyrrolidinone 
a

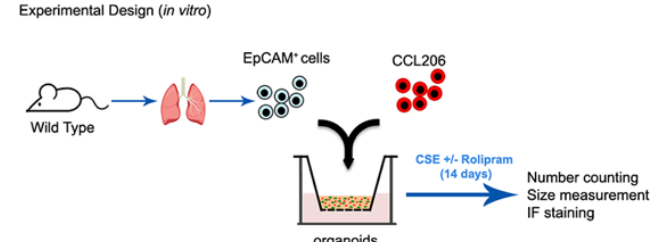

b
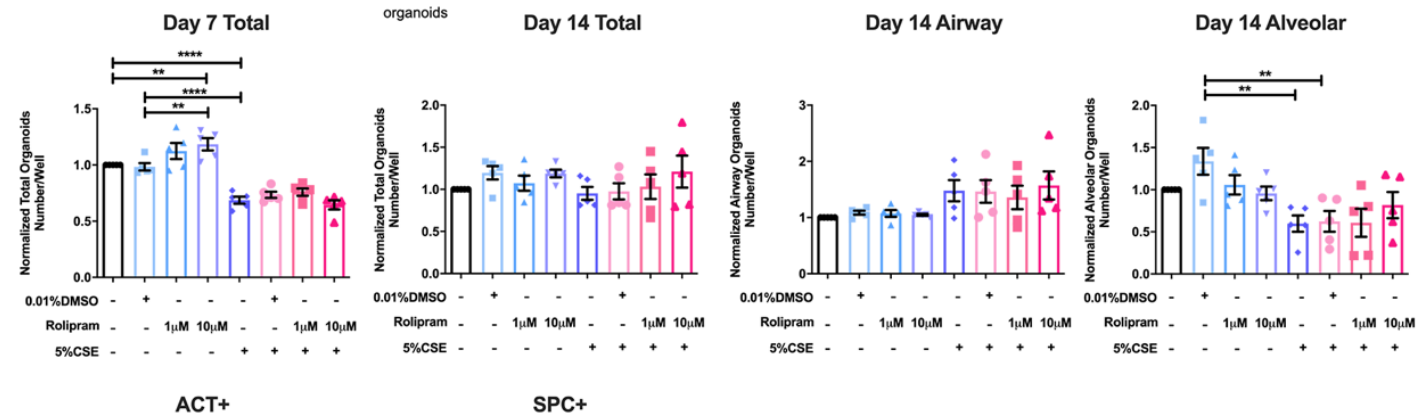

C
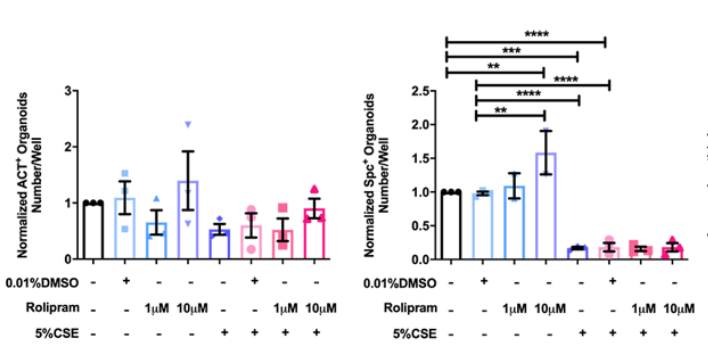

d

e

$\mathrm{f}$
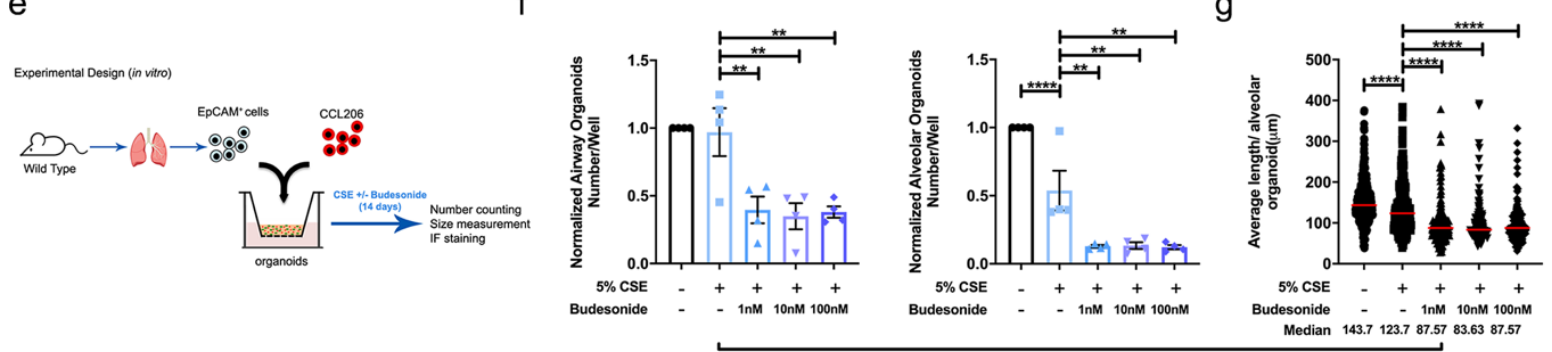

h
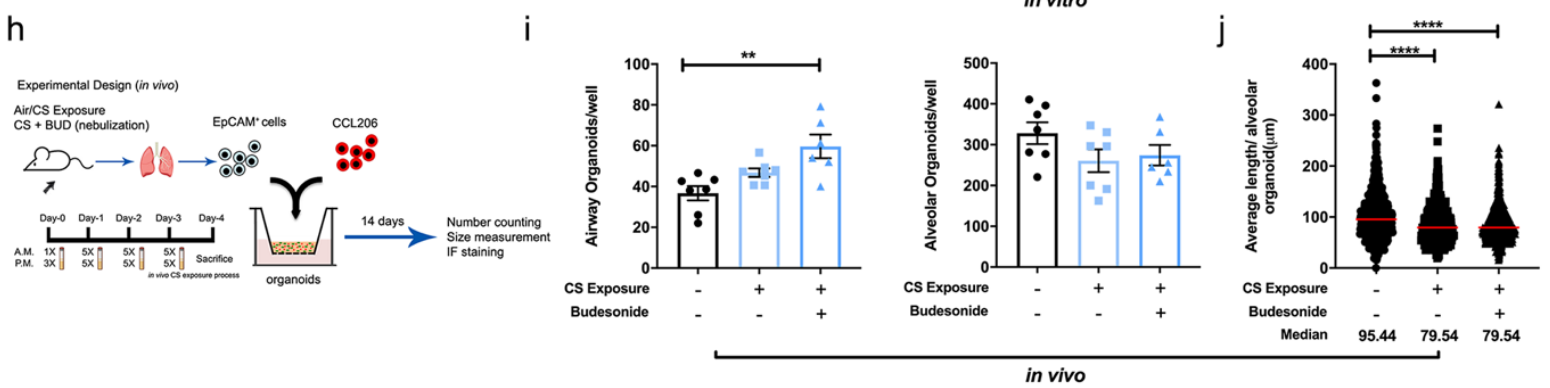

Supplementary figure 1. Effect of Rolipram and Budesonide on lung organoid formation.

a Schematic of in vitro experimental design. b Quantification of normalized number of total organoids

(day 7 ), total organoids (day 14), airway type organoids (day 14), alveolar type organoids (day 14) 
587 with medians. e Schematic of in vitro experimental design. f Quantification of normalized number of 588 airway and alveolar type organoids treated with 5\% CSE \pm Budesonide (0-, 1-, 10-, $100 \mathrm{nM})$ measured 589 on day 14. g Quantification of average length (diameter) of alveolar type organoids (median value) 590 treated with $5 \%$ CSE \pm Budesonide (0-, 1-, 10-, $100 \mathrm{nM})$ measured on day $14 . \mathrm{N}=4$ experiments, $\mathrm{n}>$ 591165 organoids/group. Data are presented as scatter plots with medians. $\mathbf{h}$ Schematic of in vivo 592 experimental design. i Number of airway and alveolar type organoids from co-culture of CCL-206

593 fibroblasts and EpCAM ${ }^{+}$cells (isolated from air-exposed, CS-exposed, and CS-exposed + Budesonide

594 nebulized mice) quantified on day 14. j Quantification of average length (diameter) of alveolar organoids

595 (median value) from co-culture of CCL-206 fibroblasts and $\mathrm{EpCAM}^{+}$cells (isolated from air-exposed,

596 CS-exposed, and CS-exposed + Budesonide nebulized mice) on day 14. $\mathrm{N}=6-7$ experiments, $\mathrm{n}>$

597671 organoids/group. Data are presented as scatter plots with medians. ${ }^{* *} p<0.05,{ }^{* *} p<0.01,{ }^{* *} p<$ $5980.001,{ }^{* * * *} p<0.0001$ 

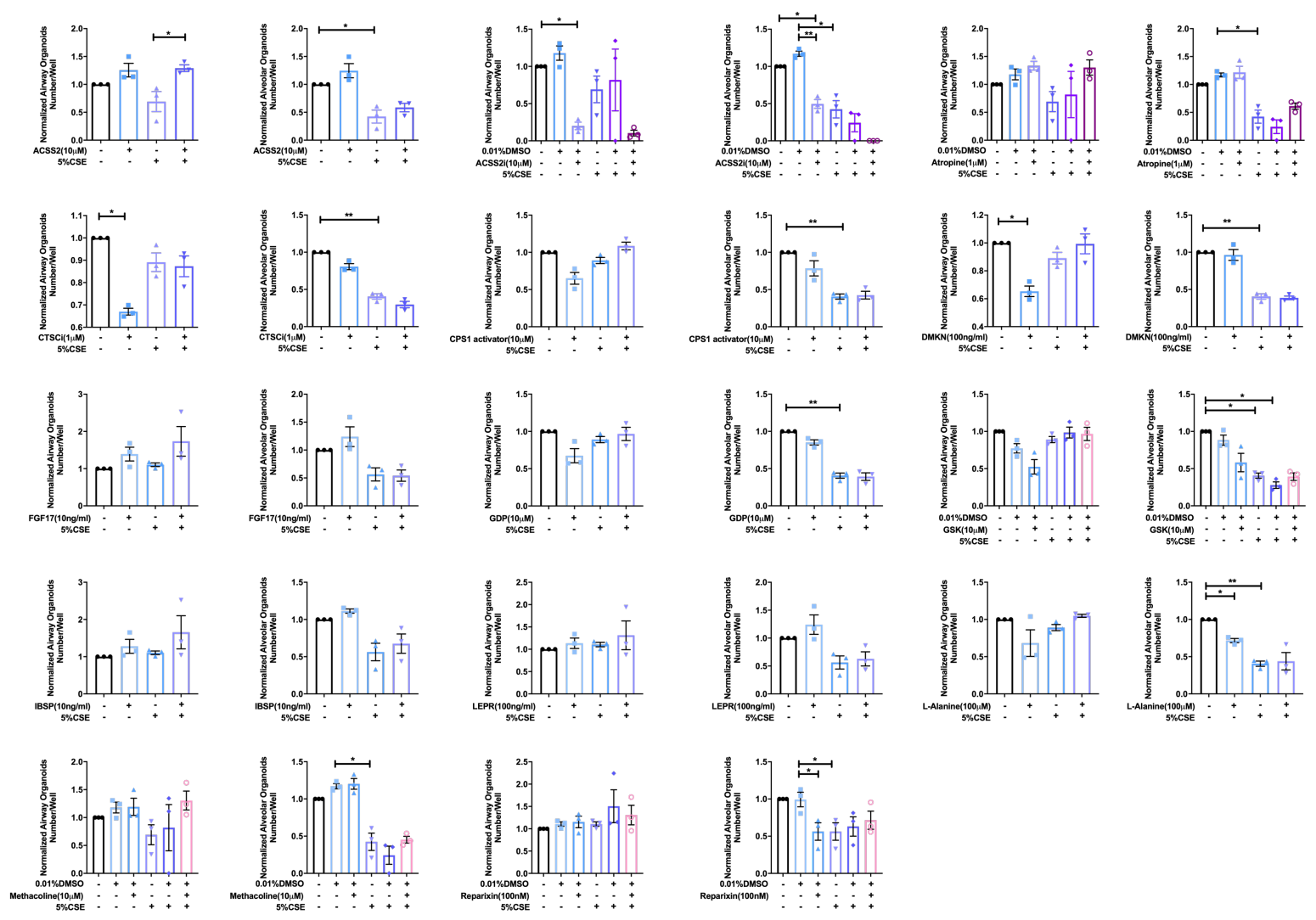

600 Supplementary figure 2 Effects of 15 drugs candidates of interest (including an additional agonist and

601 antagonist for one gene target) on the normalized number of airway and alveolar type lung organoids

602 in the presence and absence of $5 \%$ CSE. Data are presented as median \pm SEM. ${ }^{* *} p<0.05,{ }^{* *} p<0.01$, 603 ${ }^{* * *} p<0.001,{ }^{* * * *} p<0.0001$. 

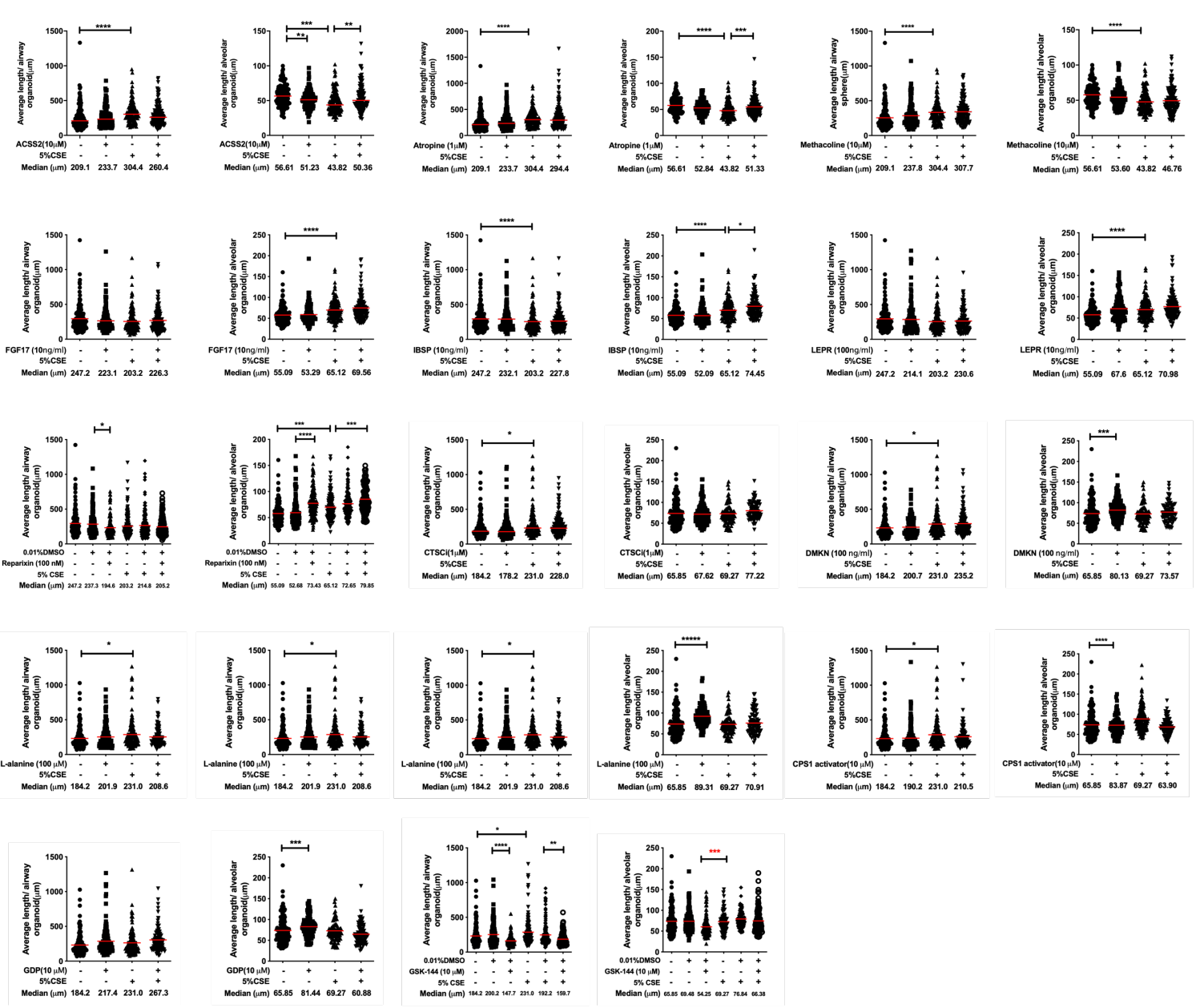

Supplementary figure 3 Effects of 15 drugs candidates of interest (including an additional agonist and antagonist for one gene target) on the size of airway and alveolar type lung organoids in the presence
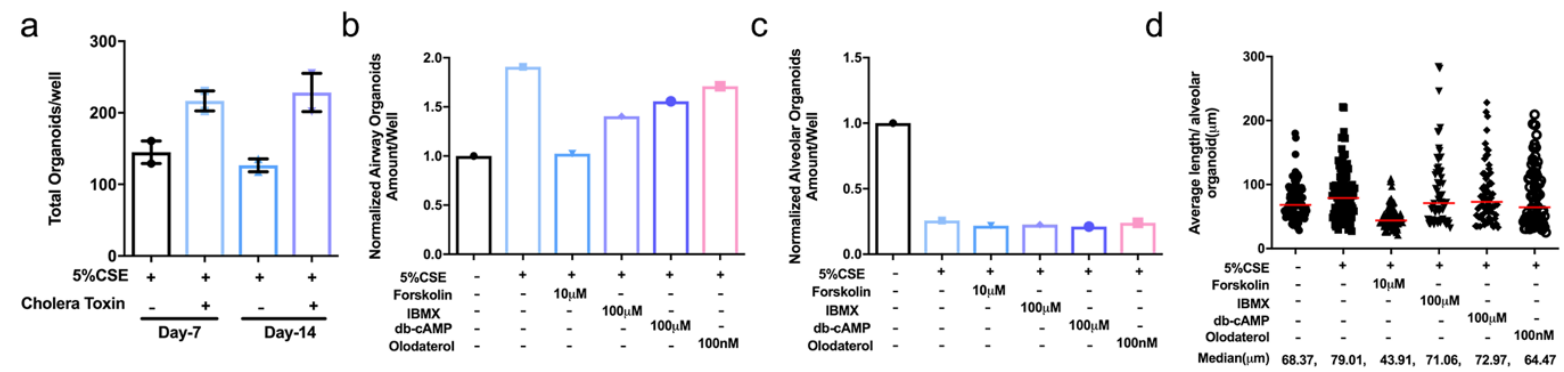

610 Supplementary figure 4 Compounds related to cAMP signaling pathways tested on lung organoid assay. a Quantification of total organoids treated with 5\% CSE + cholera toxin at different

612 time points. b Quantification of normalized airway type organoids treated with 5\% CSE \pm Forskolin (10

$613 \mu \mathrm{M})$, IBMX $(100 \mu \mathrm{M}), \mathrm{db}-\mathrm{cAMP}(100 \mu \mathrm{M})$, and olodaterol $(100 \mathrm{nM})$. c Quantification of normalized

614 alveolar type organoids treated with 5\% CSE \pm Forskolin $(10 \mu \mathrm{M}), \mathrm{IBMX}(100 \mu \mathrm{M}), \mathrm{db}-\mathrm{cAMP}(100 \mu \mathrm{M})$, 
bioRxiv preprint doi: https://doi.org/10.1101/2021.05.18.444655; this version posted May 18, 2021. The copyright holder for this preprint (which was not certified by peer review) is the author/funder. All rights reserved. No reuse allowed without permission.

615 and olodaterol (100 nM). d Quantification of average length (diameter) of alveolar type organoids

616 (median value) treated with $5 \%$ CSE \pm Forskolin $(10 \mu \mathrm{M})$, IBMX $(100 \mu \mathrm{M}), \mathrm{db}-\mathrm{CAMP}(100 \mu \mathrm{M})$, and

617 olodaterol $(100 \mathrm{nM})$. Data are presented as median \pm SEM. ${ }^{* *} \mathrm{p}<0.05,{ }^{* *} \mathrm{p}<0.01,{ }^{* * *} \mathrm{p}<0.001,{ }^{* * *} \mathrm{p}$

$618<0.0001$

619 

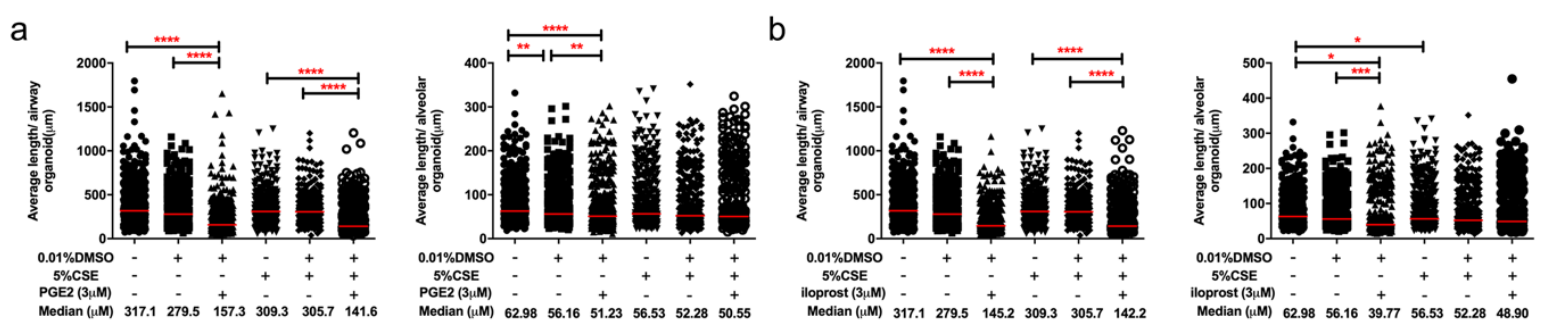

C
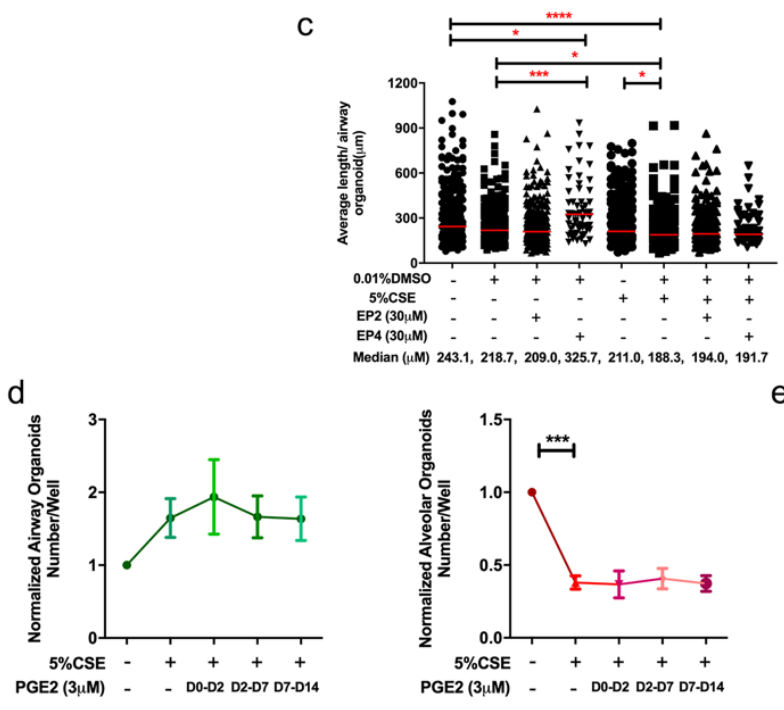

e

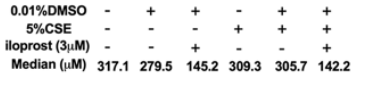

$0.01 \% \mathrm{DMSO}$
$5 \% \mathrm{CSE}$
iloprost $(3 \mu \mathrm{M})$
Median $(\mu M)$

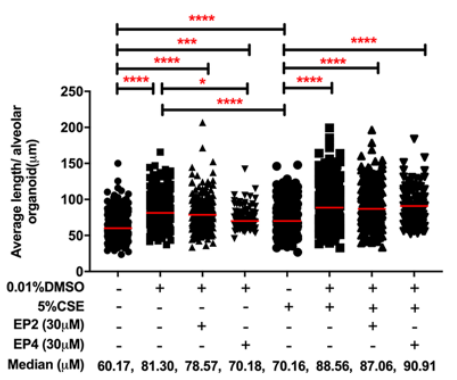

e
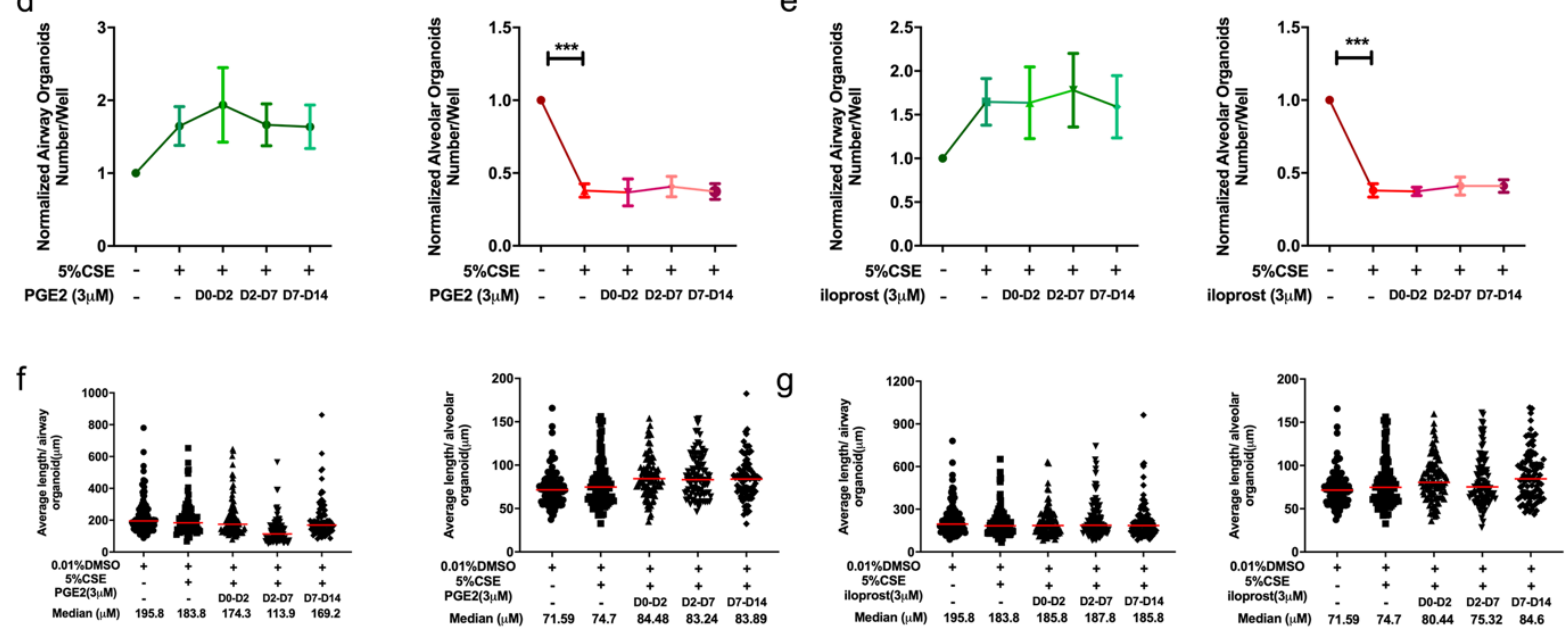

622 Supplementary figure 5 Quantification of lung organoid numbers and sizes in the study of

623 PGE2/PGI2/EP2/EP4. a-b Quantification of average length (diameter) of organoids (median value) of airway and alveolar type organoids treated with $5 \%$ CSE \pm PGE2 agonist (a)/iloprost (b) measured on day 14. $\mathrm{N}=5$ experiments, $\mathrm{n}>334$ organoids/group. Data are presented as median \pm SEM. $\mathbf{c}$

626 Quantification of average length (diameter) of airway and alveolar type organoids (median value) treated with $5 \%$ CSE \pm EP2/EP4 agonists measured on day $14 . \mathrm{N}=5$ experiments, $\mathrm{n}>264$ organoids/group. Data are presented as median \pm SEM. d-e Quantification of normalized number of airway and alveolar type organoids treated with vehicle control, 5\% CSE \pm PGE2 agonist (d)/ iloprost (e) from day 0-2, day 2-7, or day 7-14. f-g Quantification of average length (diameter) of airway and alveolar type organoids (median value) treated with 5\% CSE \pm PGE2 agonist (f)/ iloprost (g) from day 
$6320-2$, day 2-7, and day 7-14. $\mathrm{N}=2$ experiments, $\mathrm{n}>85$ organoids/group. Data are presented as median $633 \pm$ SEM.
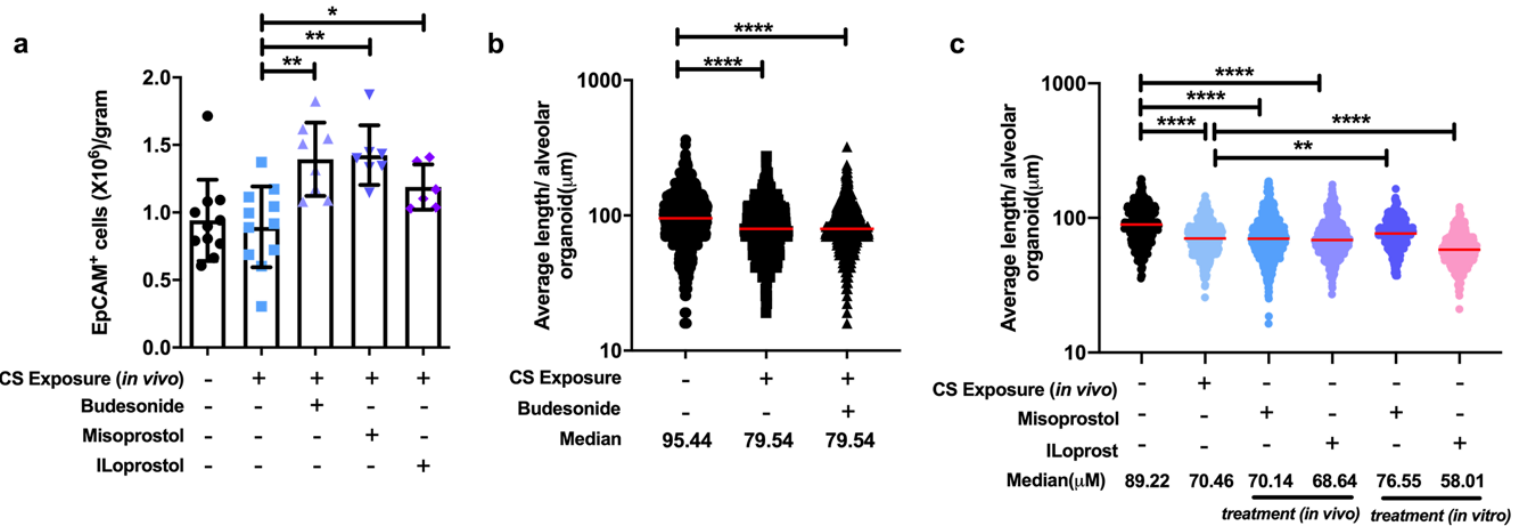

634

635 Supplementary figure 6 Quantification analysis of lung organoid assay in the in vivo study of

636 PGE2/PGI2/Budesonide. a Yield efficiency of $\mathrm{EpCAM}^{+}$cells from mice with different treatments. $\mathbf{b}$

637 Quantification of average length of alveolar type organoids co-cultured from CCL-206 and EpCAM ${ }^{+}$

638 cells isolated from air- (control) or CS-exposed mice with or without in vivo treatment with misoprostol

639 or iloprost (i.p injection). $\mathrm{N}=4-8$ experiments, $\mathrm{n}=400$ organoids/group. c Quantification of average

640 length of alveolar type organoids co-cultured from CCL-206 and EpCAM ${ }^{+}$cells isolated from air- or CS-

641 exposed mice. Organoids were treated in vitro with misoprostol or iloprost for 14 days. $\mathrm{N}=4-8$

642 experiments, $n=400$ organoids/group. Data are presented as median \pm SEM. ${ }^{* *} p<0.05,{ }^{* *} p<0.01$,

$643 \quad{ }^{* * *} p<0.001,{ }^{* * * *} p<0.0001$.

644

645 


\section{References}

647 1. Kokturk, N., Yıldırım, F., Gülhan, P. Y. \& Oh, Y. M. Stem cell therapy in chronic obstructive pulmonary disease. How far is it to the clinic? Am. J. Stem Cells 7, 56-71 (2018).

2. Basil, M. C. et al. The Cellular and Physiological Basis for Lung Repair and Regeneration: Past, Present, and Future. Cell Stem Cell 26, 482-502 (2020).

3. Volckaert, T. et al. Fgf10-Hippo Epithelial-Mesenchymal Crosstalk Maintains and Recruits Lung Basal Stem Cells. Dev. Cell 43, 48-59.e5 (2017).

4. $\mathrm{Hu}, \mathrm{Y}$. et al. Wnt/ß-catenin signaling is critical for regenerative potential of distal lung epithelial progenitor cells in homeostasis and emphysema. Stem Cells 38 (11) 1467-1478 (2020).

5. Conlon, T. M. et al. Inhibition of LT $3 R$ signalling activates WNT-induced regeneration in lung. Nature 588, 151-156 (2020).

6. Wu et al. Mesenchymal WNT-5A/5B Signaling Represses Lung Alveolar Epithelial Progenitors. Cells 8 (10), 1147 (2019).

7. Brandsma, C. A. et al. A large lung gene expression study identifying fibulin-5 as a novel player in tissue repair in COPD. Thorax 70, 21-32 (2015).

8. Kistemaker, L. E. M. et al. Muscarinic M3 receptors on structural cells regulate cigarette smoke-induced neutrophilic airway inflammation in mice. Am. J. Physiol. - Lung Cell. Mol. Physiol. 308, L96-L103 (2015).

9. Ng-Blichfeldt, J. P. et al. Retinoic acid signaling balances adult distal lung epithelial progenitor cell growth and differentiation. EBioMedicine 36, 461-474 (2018).

10. Woodby, B. et al. The PDE4 inhibitor CHF6001 affects keratinocyte proliferation via cellular redox pathways. Arch. Biochem. Biophys. 685, 1-8 
(2020).

672 11. Zuo, H. et al. Cigarette smoke up-regulates PDE3 and PDE4 to decrease cAMP in airway cells. Br. J. Pharmacol. 175, 2988-3006 (2018).

12. Kothe, T. B. et al. Surfactant and budesonide for respiratory distress syndrome: an observational study. Pediatr. Res. 87, 940-945 (2020).

13. Ricci, F. et al. In vitro and in vivo characterization of poractant alfa supplemented with budesonide for safe and effective intratracheal administration. Pediatr. Res. 82, 1056-1063 (2017).

14. Tashkin, D. P., Lipworth, B. \& Brattsand, R. Benefit:Risk Profile of Budesonide in Obstructive Airways Disease. Drugs 79, 1757-1775 (2019).

15. Waters, R. C. \& Hochhaus, G. Characterization of a dextran-budesonide prodrug for inhalation therapy. Eur. J. Pharm. Sci. 129, 58-67 (2019).

16. Shen, W. et al. SR9009 induces a REV-ERB dependent anti-small-cell lung cancer effect through inhibition of autophagy. Theranostics 10, 4466-4480 (2020).

17. Durrington, H. J. et al. Circadian asthma airway responses are gated by REVERBa. Eur. Respir. J. 56, 1-13 (2020).

18. Sundar, I. K., Rashid, K., Sellix, M. T. \& Rahman, I. The nuclear receptor and clock gene REV-ERBa regulates cigarette smoke-induced lung inflammation. Biochem. Biophys. Res. Commun. 493, 1390-1395 (2017).

19. Gindele, J. A. et al. Intermittent exposure to whole cigarette smoke alters the differentiation of primary small airway epithelial cells in the air-liquid interface culture. Sci. Rep. 10, 1-17 (2020). under metabolic stress. Br. J. Cancer 120, 1090-1098 (2019). 
21. Gao, X. et al. Acetate functions as an epigenetic metabolite to promote lipid synthesis under hypoxia. Nat. Commun. 7, (2016).

22. Whyte, M. P. et al. Hypophosphatemic osteosclerosis, hyperostosis, and enthesopathy associated with novel homozygous mutations of DMP1 encoding dentin matrix protein 1 and SPP1 encoding osteopontin: The first digenic SIBLING protein osteopathy? Bone 132, 115190 (2020).

23. Shintani, S. et al. Identification and characterization of integrin-binding sialoprotein (IBSP) genes in reptile and amphibian. Gene 424, 11-17 (2008).

24. Zhang, L. et al. Predictive significance of bone sialoprotein and osteopontin for bone metastases in resected Chinese non-small-cell lung cancer patients: A large cohort retrospective study. Lung Cancer 67, 114-119 (2010).

25. Guo, X. et al. Leptin signaling in intestinal epithelium mediates resistance to enteric infection by Entamoeba histolytica. Mucosal Immunol. 4, 294-303 (2011).

26. Daniel E Shumer, N. J. N. N. P. S. Leptin receptor polymorphisms and lung function decline in COPD. Physiol. Behav. 176, 139-148 (2017).

27. Lee, K., Lee, S. H. \& Kim, T. H. The biology of prostaglandins and their role as a target for allergic airway disease therapy. Int. J. Mol. Sci. 21, (2020).

28. Take, Y., Koizumi, S. \& Nagahisa, A. Prostaglandin E Receptor 4 Antagonist in Cancer Immunotherapy: Mechanisms of Action. Front. Immunol. 11, 1-7 (2020).

29. Dey, I., Lejeune, M. \& Chadee, K. Prostaglandin E 2 receptor distribution and function in the gastrointestinal tract. Br. J. Pharmacol. 149, 611-623 (2006).

30. Markovič, T., Jakopin, Ž., Dolenc, M. S. \& Mlinarič-Raščan, I. Structural features of subtype-selective EP receptor modulators. Drug Discov. Today 22, 
57-71 (2017).

722

723

724

725

726

727

728

729

730

731

732

733

734

735

736

737

738

739

740

741

742

743

744

745

31. Nunez, F. J. et al. Agonist-specific desensitization of PGE2-stimulated cAMP signaling due to upregulated phosphodiesterase expression in human lung fibroblasts. Naunyn. Schmiedebergs. Arch. Pharmacol. 393, 843-856 (2020).

32. Tilley, S. L. et al. Receptors and pathways mediating the effects of prostaglandin E2 on airway tone. Am. J. Physiol. - Lung Cell. Mol. Physiol. 284, 599-606 (2003).

33. Buckley, J. et al. EP4 receptor as a new target for bronchodilator therapy. Thorax 66, 1029-1035 (2011).

34. Birrell, M. A. et al. Anti-inflammatory effects of PGE2 in the lung: Role of the EP4 receptor subtype. Thorax 70, 740-747 (2015).

35. Wang, L. et al. Hemodynamic and gas exchange effects of inhaled iloprost in patients with COPD and pulmonary hypertension. Int. J. COPD 12, 3353-3360 (2017).

36. Zhu, Y. et al. A prostacyclin analogue, iloprost, protects from bleomycininduced pulmonary fibrosis in mice. Respir. Res. 11, 1-12 (2010).

37. Gohrbandt, B. et al. lloprost to improve surfactant function in porcine pulmonary grafts stored for twenty-four hours in low-potassium dextran solution. J. Thorac. Cardiovasc. Surg. 129, 80-86 (2005).

38. Kim, N. et al. Effects of Inhaled Iloprost on Lung Mechanics and Myocardial Function During One-Lung Ventilation in Chronic Obstructive Pulmonary Disease Patients Combined With Poor Lung Oxygenation. Anesth. Analg. 130 (5), 1407-1414 (2020).

39. Lee, S. H. et al. Effects of intraoperative inhaled iloprost on primary graft dysfunction after lung transplantation: A retrospective single center study. 
Med. 95 (27), (2016).

747

40. Gallardo, A. et al. The molecular clock protein Bmal1 regulates cell differentiation in mouse embryonic stem cells. Life Sci. alliance 3, 1-12 (2020).

41. Almeida, S., Chaves, M. \& Delaunay, F. Transcription-based circadian mechanism controls the duration of molecular clock states in response to signaling inputs. J. Theor. Biol. 484, 1-11 (2020).

42. Braghiroli, A. et al. Day and night control of copd and role of pharmacotherapy: A review. Int. J. COPD 15, 1269-1285 (2020).

43. Zou, X. et al. A Systems Biology Approach Identifies Hidden Regulatory Connections Between the Circadian and Cell-Cycle Checkpoints. Front. Physiol. 11, 1-9 (2020).

44. Gaucher, J., Montellier, E. \& Sassone-Corsi, P. Molecular Cogs: Interplay between Circadian Clock and Cell Cycle. Trends Cell Biol. 28, 368-379 (2018).

45. Rahman, I. Antioxidant therapies in COPD. Int. J. Chron. Obstruct. Pulmon. Dis. 1, 15-29 (2006).

46. Yu, D. et al. Rev-erba can regulate the NF-KB/NALP3 pathway to modulate lipopolysaccharide-induced acute lung injury and inflammation. Int. Immunopharmacol. 73, 312-320 (2019).

47. Wiman, K. G. \& Zhivotovsky, B. Understanding cell cycle and cell death regulation provides novel weapons against human diseases. J. Intern. Med. 281, 483-495 (2017).

48. Maddika, S. et al. Cell survival, cell death and cell cycle pathways are interconnected: Implications for cancer therapy. Drug Resist. Updat. 10, 13-29 (2007). 
49. Padgett, J. \& Santos, S. D. M. From clocks to dominoes: lessons on cell cycle remodelling from embryonic stem cells. FEBS Lett. 594, 2031-2045 (2020).

50. Farshadi, E., van der Horst, G. T. J. \& Chaves, I. Molecular Links between the Circadian Clock and the Cell Cycle. J. Mol. Biol. 3515-3524 (2020).

51. Zepp, J. A. \& Morrisey, E. E. Cellular crosstalk in the development and regeneration of the respiratory system. Nat. Rev. Mol. Cell Biol. 20, 551-566 (2019).

52. Almeida, S., Chaves, M. \& Delaunay, F. Control of synchronization ratios in clock/cell cycle coupling by growth factors and glucocorticoids. $R$. Soc. Open Sci. 7, (2020).

53. Abraham, U. et al. Coupling governs entrainment range of circadian clocks. Mol. Syst. Biol. 6, 1-13 (2010).

54. Kistemaker, L. E. M. et al. Tiotropium attenuates IL-13-induced goblet cell metaplasia of human airway epithelial cells. Thorax 70, 668-676 (2015).

55. Morrow, J. D. et al. Functional interactors of three genome-wide association study genes are differentially expressed in severe chronic obstructive pulmonary disease lung tissue. Sci. Rep. 7, 1-11 (2017).

56. Ng-Blichfeldt, J. P. et al. Tgf- $\beta$ activation impairs fibroblast ability to support adult lung epithelial progenitor cell organoid formation. Am. J. Physiol. - Lung Cell. Mol. Physiol. 317 (1), L14-L28, (2019).

57. Torre, D., Lachmann, A. \& Ma'ayan, A. BioJupies: Automated Generation of Interactive Notebooks for RNA-Seq Data Analysis in the Cloud. Cell Syst. 7, 556-561.e3 (2018). 